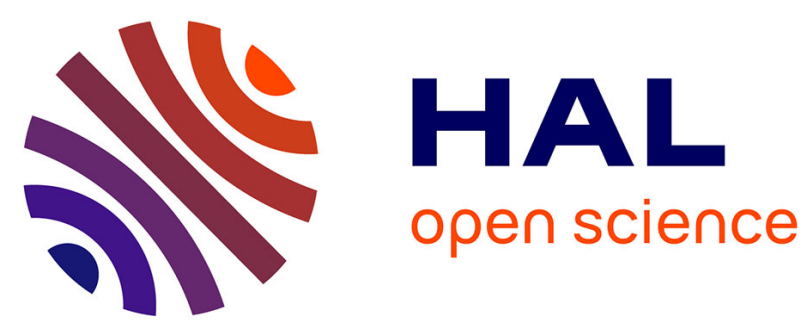

\title{
Side-sensitive modified group runs charts with and without measurement errors for monitoring the coefficient of variation
}

\author{
Sajal Saha, Michael B C Khoo, Philippe Castagliola, Abdul Haq
}

\section{To cite this version:}

Sajal Saha, Michael B C Khoo, Philippe Castagliola, Abdul Haq. Side-sensitive modified group runs charts with and without measurement errors for monitoring the coefficient of variation. Quality and Reliability Engineering International, 2021, 37 (2), pp.598-617. 10.1002/qre.2751 . hal-03321793

\section{HAL Id: hal-03321793 \\ https://hal.science/hal-03321793}

Submitted on 18 Aug 2021

HAL is a multi-disciplinary open access archive for the deposit and dissemination of scientific research documents, whether they are published or not. The documents may come from teaching and research institutions in France or abroad, or from public or private research centers.
L'archive ouverte pluridisciplinaire HAL, est destinée au dépôt et à la diffusion de documents scientifiques de niveau recherche, publiés ou non, émanant des établissements d'enseignement et de recherche français ou étrangers, des laboratoires publics ou privés. 
Side-sensitive modified group runs charts with and without measurement errors for monitoring the coefficient of variation

\author{
Sajal Saha \\ Department of Mathematics, \\ International University of Business Agriculture and Technology, Dhaka, Bangladesh \\ sajal.saha@iubat.edu
}

Michael B. C. Khoo (Corresponding Author)

School of Mathematical Sciences, Universiti Sains Malaysia, 11800 Penang, Malaysia mkbc@usm.my

Philippe Castagliola

Université de Nantes \& LS2N UMR CNRS 6004, Nantes, France philippe.castagliola@univ-nantes.fr

\author{
Abdul Haq \\ Department of Statistics, Quaid-i-Azam University, Islamabad, Pakistan \\ aaabdulhaq@yahoo.com
}




\title{
Side-sensitive modified group runs charts with and without measurement errors for monitoring the coefficient of variation
}

\begin{abstract}
The coefficient of variation (CV) is used in process monitoring when the process mean and standard deviation are proportional to each other. In this work, a side-sensitive modified group runs $\mathrm{CV}$ (SSMGR CV) chart is proposed for monitoring the process $\mathrm{CV}$. The run length performance of the SSMGR CV chart is compared with those of the existing CV charts in terms of the average and standard deviation of the run length criteria. The SSMGR CV chart is found to outperform the existing CV charts. In addition, the run length performance of the SSMGR CV chart is also evaluated in the presence of measurement errors, as these errors are not only unavoidable in practice but they also affect the sensitivity of a control chart in detecting an out-ofcontrol situation. The results obtained show that the accuracy and precision errors affect the performance of the SSMGR CV chart in detecting an out-of-control situation.
\end{abstract}

Keywords: Coefficient of variation; side-sensitive modified group runs (SSMGR); average run length; standard deviation of the run length; measurement error

\section{Introduction}

A control chart is the most important tool in Statistical Process Control (SPC) to detect process shifts, in order to reduce process variability in manufacturing or service industries. The $\bar{X}$ and $S$ charts are commonly used to ensure that the process mean and standard deviation, respectively, remain in-control at their nominal values, $\mu_{0}$ and $\sigma_{0}$, respectively. However, there are many 
processes where the mean is not constant and/or the standard deviation is a function of the mean. In such processes, the process mean and standard deviation may change but as long as the ratio of the process standard deviation to the mean is constant, the said processes are assumed to be incontrol. For this type of processes, the conventional $\bar{X}$ and $S$ charts are no longer suitable in process monitoring because these two charts monitor the process mean and process standard deviation separately and neither of them monitors the ratio of the process standard deviation to the mean. Instead, the $\mathrm{CV}$ (denoted as $\gamma=\sigma / \mu$ ), which is a ratio of the process standard deviation to the mean, is a suitable quality characteristic in process monitoring for the aforesaid type of processes.

Kang et al ${ }^{1}$ introduced the Shewhart CV chart and showed that this chart is an effective quality control tool for quality improvement, when neither the process mean nor the process variance is constant. However, the Shewhart CV chart is not efficient in detecting small and moderate CV shifts. To improve the efficiency of the Shewhart CV chart, Hong et al ${ }^{2}$ proposed a two-sided exponentially weighted moving average (EWMA) CV chart that is efficient in detecting small and moderate CV shifts. Later, Castagliola et al ${ }^{3}$ presented a one-sided EWMA CV chart and showed that the new chart is more efficient than the two-sided EWMA CV chart of Hong et al ${ }^{2}$ in detecting CV shifts. To incorporate adaptive features into the Shewhart CV chart, Castagliola et al ${ }^{4}$ and Castagliola et al ${ }^{5}$ proposed the variable sampling interval $\mathrm{CV}$ and variable sample size $\mathrm{CV}$ charts, respectively, where they found that these CV charts outperform the Shewhart CV chart.

On similar lines, Calzada and Scariano ${ }^{6}$ proposed the synthetic CV chart and compared its performance with the Shewhart CV and one-sided EWMA CV charts. The synthetic CV chart was found to outperform the Shewhart CV chart but the EWMA CV chart is superior to the synthetic $\mathrm{CV}$ chart in detecting small $\mathrm{CV}$ shifts. You et al ${ }^{7}$ studied the performance of the side-sensitive 
group runs (SSGR) chart for monitoring the $\mathrm{CV}$ and compared its performance with the Shewhart $\mathrm{CV}$, synthetic CV and EWMA CV charts. The findings showed that the SSGR CV chart surpasses the basic $\mathrm{CV}$ and synthetic $\mathrm{CV}$ charts. Noor-ul-Amin et al ${ }^{8}$ proposed a blended control chart with and without auxiliary information for a simultaneous monitoring of the process mean and $\mathrm{CV}$ and found that the sensitivity of the proposed chart is enhanced by incorporating auxiliary information. An EWMA chart for multivariate CV was introduced by Giner-Bosch ${ }^{9}$ and it was shown that the aforementioned chart outperforms its existing counterparts. For recent research works on the CV charts, readers may refer to Teoh et al, ${ }^{10}$ Khaw et al, ${ }^{11}$ Yeong et al, ${ }^{12}$ Yeong et al ${ }^{13}$ and Chew et al $^{14}$

Numerous group runs (GR) schemes were introduced to enhance the sensitivity of the synthetic $\bar{X}$ chart proposed by Wu and Spedding ${ }^{15}$. This paragraph discusses the GR schemes that exist in the literature. Gadre and Rattihalli ${ }^{16}$ introduced the GR $\bar{X}$ chart by combining the $\bar{X}$ chart with the conforming run length (CRL) chart. The CRL sub-chart is a type of lower-sided chart that is used to determine whether the process being monitored is in-control or otherwise. The GR $\bar{X}$ chart signals an out-of-control if $\mathrm{CRL}_{1}<L$ or both $\mathrm{CRL}_{r}$ and $\mathrm{CRL}_{r+1}$ (for $r>1$ ) are less than $L$, where $L$ is the lower limit of the CRL sub-chart (of the GR $\bar{X}$ chart). Here, $\mathrm{CRL}_{r}$ represents the number of conforming samples, inspected between the $(r-1)^{\text {th }}$ and $r^{\text {th }}$ nonconforming samples, including the $r^{\text {th }}$ non-conforming sample. It was found that the GR $\bar{X}$ chart outperforms the synthetic $\bar{X}$ and Shewhart $\bar{X}$ charts.

Gadre and Rattihalli ${ }^{17}$ extended the GR $\bar{X}$ charting concept to the modified GR (MGR) $\bar{X}$ chart by adding an additional lower limit to the CRL sub-chart (of the MGR $\bar{X}$ chart). The MGR $\bar{X}$ chart signals an out-of-control if $\mathrm{CRL}_{1}<L_{2}$ or for some $r(>1), \mathrm{CRL}_{r}<L_{1}$ and $\mathrm{CRL}_{r+1}<L_{2}$. 
Here, $L_{1}$ and $L_{2}$ are the lower limits of the CRL sub-chart (of the MGR $\bar{X}$ chart). Note that if $L_{1}=L_{2}=L$, the MGR $\bar{X}$ chart reduces to the GR $\bar{X}$ chart. On similar lines, by adding the sidesensitive feature to the GR $\bar{X}$ and MGR $\bar{X}$ charts, Gadre and Rattihalli ${ }^{18}$ and Gadre et al ${ }^{19}$ introduced the side-sensitive GR (SSGR) $\bar{X}$ and the side-sensitive MGR (SSMGR) $\bar{X}$ charts, respectively. Both the SSGR and SSMGR charts signal an out-of-control if the sample means $(\bar{X})$ corresponding to the two successive CRLs that contribute to the out-of-control signal plot on the same side of the target value on the $\bar{X}$ sub-chart (of the respective SSGR $\bar{X}$ and SSMGR $\bar{X}$ charts). In terms of the effectiveness of the charts in detecting process mean shifts, Gadre et al ${ }^{19}$ showed that the SSMGR $\bar{X}$ chart outperforms the Shewhart $\bar{X}$, synthetic $\bar{X}$, GR $\bar{X}$, MGR $\bar{X}$ and SSGR $\bar{X}$ charts. More recent extensions on the GR type charts include You et al, ${ }^{20}$ Khoo et al, ${ }^{21}$ Chong et al, ${ }^{22}$ Saha et al, ${ }^{23}$ Chong et al, ${ }^{24}$ Mim et al, ${ }^{25}$ and Gadre and Kakade. ${ }^{26}$

Note that in the Shewhart $\bar{X}$ chart, a decision about the state of the process being monitored is dependent on where the sample mean plots on the chart. If the sample mean plots beyond the limits of the $\bar{X}$ chart, the process is out-of-control, otherwise, it is in-control. On the other hand, for the $\bar{X}$ sub-chart of a GR $\bar{X}$ type chart (such as the MGR $\bar{X}$, SSGR $\bar{X}$ and SSMGR $\bar{X}$ charts), a sample mean that plots beyond (within) the limits of the $\bar{X}$ sub-chart is considered as a non-conforming (conforming) sample, instead of an out-of-control (in-control) sample. A decision as to whether a process being monitored using a GR $\bar{X}$ type chart is in-control or out-of-control is made according to the outcome given by the conforming run length (CRL) sub-chart, where the points on the CRL sub-chart are plotted based on the information provided by the $\bar{X}$ sub-chart.

Control charts are usually designed with the assumption that the measurements on the quality characteristics are obtained without any measurement error. However, in usual practice, 
measurement errors often exist and affect the performances of the control charts. Linna and Woodall ${ }^{27}$ introduced the linear covariate error model to investigate the effect of measurement errors on the $\bar{X}$ and $S^{2}$ charts, and they recommended taking multiple measurements per item in order to reduce the effect of measurement errors. Using the same covariate error model of Linna and Woodall, ${ }^{27}$ Linna et al ${ }^{28}$ studied the performance of multivariate control charts in the presence of measurement errors, where they found that the ability of the control charts to detect shifts in one direction is better than in the other direction due to the loss of the directional invariance property. Costa and Castagliola ${ }^{29}$ studied the performance of the $\bar{X}$ chart in the presence of measurement errors and autocorrelated data, and they showed that the effect of autocorrelation can be reduced by taking samples with non-neighboring items. The effects of measurement errors on the two one-sided Shewhart charts for monitoring the ratio of two normal variables were investigated by Nguyen and Tran ${ }^{30}$, where it was found that the two one-sided charts are more advantageous than the two-sided Shewhart chart for the ratio. Additionally, Tran et al ${ }^{31}$ proposed the synthetic median chart to improve the efficiency of the Shewhart median chart in detecting small and moderate mean shifts, followed by conducting an investigation of the effects of measurement errors on the synthetic median chart. Additional researches that investigated the effect of measurement errors on different types of control charts were made by Maravelakis, ${ }^{32} \mathrm{Hu}$ et al, ${ }^{33}$ Noorossana and Zerehsaz, ${ }^{34}$ Tran et al, ${ }^{35}$ Tran et al, ${ }^{36}$ Yeong et al ${ }^{37}$ and Tran et al. ${ }^{38}$

Due to the sensitivity of the SSMGR chart towards process shifts and the widespread use of $\mathrm{CV}$ in real life, the SSMGR chart for monitoring the process CV (called the SSMGR CV chart) is developed in this research. The charting statistic, optimal design and implementation procedure of the proposed chart are presented. The SSMGR CV chart is compared with the existing EWMA $\mathrm{CV}$, run sum (RS) CV and SSGR CV charts, in terms of the average run length (ARL) and standard 
deviation of the run length (SDRL) criteria, where the results show that the SSMGR CV chart generally outperforms the existing charts. Additionally, the detection ability of the SSMGR CV chart in the presence of measurement errors (called the SSMGR CV-ME chart) is also investigated in detail. The charting statistic, optimal design and implementation procedure of the SSMGR CVME chart are also discussed. Moreover, a step-by-step implementation procedure of the SSMGR CV-ME chart using a real industrial dataset is given to explain the working of the proposed chart in usual practice.

The rest of this paper is organized as follows: Section 2 presents the basic properties of the CV. The SSMGR CV chart is presented in Section 3. Section 4 explains the linear covariate error model for the CV. Section 5 discusses the SSMGR CV-ME chart. The optimal design procedure to minimize the out-of-control value of ARL for the SSMGR CV and SSMGR CV-ME charts are discussed in Section 6. The performances of the SSMGR CV and SSMGR CV-ME charts are evaluated in Section 7. Section 8 shows the implementation of the SSMGR CV-ME chart using a real dataset. Lastly, conclusions are drawn in Section 9.

\section{Basic properties of $\mathrm{CV}$}

Let $X$ be a random variable having mean $\mu$ and standard deviation $\sigma$, then the $\mathrm{CV}$ of $X$ is

$$
\gamma=\frac{\sigma}{\mu}
$$

Let $\left\{X_{1}, X_{2}, \ldots, X_{n}\right\}$ be a random sample of size $n$ from the normal $N\left(\mu, \sigma^{2}\right)$ distribution, i.e. $X_{i} \sim N\left(\mu, \sigma^{2}\right)$ for $i=1,2, \ldots, n$. The sample mean $\bar{X}$ and sample standard deviation $S$ computed from this sample are 


$$
\bar{X}=\frac{1}{n} \sum_{i=1}^{n} X_{i}
$$

and

$$
S=\sqrt{\frac{1}{n-1} \sum_{i=1}^{n}\left(X_{i}-\bar{X}\right)^{2}},
$$

respectively. Based on $\bar{X}$ and $S$, the sample $\mathrm{CV}(\hat{\gamma})$ is computed as

$$
\hat{\gamma}=\frac{S}{\bar{X}}
$$

In the literature, the probability distribution of $\hat{\gamma}$ has been investigated by numerous researchers, such as McKay, ${ }^{39}$ Iglewicz et al ${ }^{40}$ and Iglewicz and Myers, ${ }^{41}$ to name a few. Iglewicz et al ${ }^{40}$ showed that $\sqrt{n} / \hat{\gamma}$ follows the non-central $t$ distribution with $n-1$ degrees of freedom with the non-centrality parameter $\sqrt{n} / \gamma$. Following this result, Castagliola et al ${ }^{3}$ showed that the cumulative distribution function (cdf) of $\hat{\gamma}$ is

$$
F_{\hat{\gamma}}(x \mid n, \gamma)=1-F_{t}\left(\frac{\sqrt{n}}{x} \mid n-1, \frac{\sqrt{n}}{\gamma}\right)
$$

where $F_{t}\left(\cdot \mid n-1, \frac{\sqrt{n}}{\gamma}\right)$ is the cdf of the non-central $t$ distribution with $n-1$ degrees of freedom and non-centrality parameter $\sqrt{n} / \gamma$. The inverse cdf of $\hat{\gamma}$ is given by (Castagliola et al ${ }^{3}$ )

$$
F_{\hat{\gamma}}^{-1}(x \mid n, \gamma)=\frac{\sqrt{n}}{F_{t}^{-1}\left(1-x \mid n-1, \frac{\sqrt{n}}{\gamma}\right)} .
$$

\section{The SSMGR CV chart}


The SSMGR CV chart consists of the CV sub-chart and an extended version of the CRL sub-chart. The upper and lower control limits (UCL and LCL) of the CV sub-chart of the SSMGR CV chart are

$$
\mathrm{UCL}=F_{\hat{\gamma}}^{-1}\left(1-\frac{k}{2} \mid n, \gamma_{0}\right)
$$

and

$$
\mathrm{LCL}=F_{\hat{\gamma}}^{-1}\left(\frac{k}{2} \mid n, \gamma_{0}\right)
$$

respectively, where $k$ is the limits' constant that is used to adjust the limits in order to attain the desired in-control performance of the chart, while $\gamma_{0}$ is the target value of the process CV.

If the charting statistic of the SSMGR CV chart, i.e. $\hat{\gamma}$, computed using Equation (4), falls beyond the $\mathrm{UCL} / \mathrm{LCL}$ limits, the current sample $\mathrm{CV}$ is declared as non-conforming. Let $\mathrm{CRL}_{r}$ represent the number of conforming $\hat{\gamma}$ samples, inspected between the $(r-1)^{\text {th }}$ and $r^{\text {th }}$ nonconforming $\hat{\gamma}$ samples, including the $r^{\text {th }}$ non-conforming $\hat{\gamma}$ sample. The implementation of the SSMGR CV chart is as follows:

Step 1. Take a sample of size $n$ and compute $\hat{\gamma}$.

Step 2. If $\hat{\gamma} \in[\mathrm{LCL}, \mathrm{UCL}]$, the sample is classified as conforming, then return to Step 1. Otherwise, the sample is non-conforming and proceed to Step 3.

Step 3. Compute $\mathrm{CRL}_{r}$, for $r=1,2, \ldots$ If $\mathrm{CRL}_{1} \leq C_{2}$ or for $r>1, \mathrm{CRL}_{r} \leq C_{1}$ and $\mathrm{CRL}_{r+1} \leq C_{2}$, declare the process as out-of-control, where $C_{f}($ for $f=1,2)$ are the lower limits of the CRL sub-chart (of the SSMGR CV chart). Otherwise, return to Step 1. There are three design parameters of the SSMGR CV chart, i.e. $k, C_{1}$ and $C_{2}$. 
Let $P$ denote the probability of $\hat{\gamma}$ falling beyond the UCL/LCL limits of the SSMGR CV chart. Then

$$
\begin{aligned}
P & =1-\operatorname{Pr}(\mathrm{LCL} \leq \hat{\gamma} \leq \mathrm{UCL}) \\
& =1-F_{\hat{\gamma}}(\mathrm{UCL} \mid n, \gamma)+F_{\hat{\gamma}}(\mathrm{LCL} \mid n, \gamma) .
\end{aligned}
$$

As the SSMGR CV chart considers the side-sensitive feature, it is necessary to consider the conditional probability

$$
\begin{aligned}
\alpha & =\operatorname{Pr}(\hat{\gamma}>\mathrm{UCL} \mid \hat{\gamma} \notin[\mathrm{LCL}, \mathrm{UCL}]) \\
& =\frac{\operatorname{Pr}(\hat{\gamma}>\mathrm{UCL} \cap \hat{\gamma} \notin[\mathrm{LCL}, \mathrm{UCL}])}{\operatorname{Pr}(\hat{\gamma} \notin[\mathrm{LCL}, \mathrm{UCL}])} \\
& =\frac{1-F_{\hat{\gamma}}(\mathrm{UCL} \mid n, \gamma)}{P}
\end{aligned}
$$

The Markov chain approach, similar to that in Gadre et al ${ }^{19}$, is used to compute the ARL values of the SSMGR CV chart. The description of the Markov chain states of the samples are given in Table 1. Note that the states of the Markov chain model for the SSMGR CV chart depend on the values of the positive integers, say $C_{1}$ and $C_{2}$. As an example, Table 2 shows the complete states of the Markov chain model for the SSMGR CV chart when $C_{1}=C_{2}=3$. The following steps explain the procedure to obtain the states of the Markov chain model for the SSMGR CV chart:

1. A sequence starting with $\underline{\bar{G}}$ (or $\bar{G}$ or $\underline{G}$ ), followed by a maximum of $\left(C_{2}-1\right) \tilde{0}$ 's. There are $3 C_{2}$ such states (see states $1-3,5,6,8-11$ in Table 2).

2. A sequence of $C_{2} \tilde{0}$ 's. There is only one state of this type (see state 4 in Table 2). 
3. A sequence starting with $G$ ( or $\bar{H}$ or $\underline{H}$ ), followed by a maximum of $\left(C_{1}-1\right) 0$ 's. There are $3 C_{1}$ such states (see states $12-20$ in Table 2).

4. A sequence of $C_{1} 0$ 's. Only one state of this type is available (see state 7 in Table 2).

5. Signal (see state 21 in Table 2).

The total number of states, including the absorbing state is $3 C_{2}+1+3 C_{1}+1+1=$ $3\left(C_{2}+C_{1}+1\right)$. Table 2 presents the 21 different states of the Markov chain model for the SSMGR CV chart, based on $C_{1}=3$ and $C_{2}=3$. In Table 2, states $1-20$ are non-absorbing states while state 21 is an absorbing state.

Let $\boldsymbol{R}$ be the transition probability matrix (tpm) of the Markov chain model for the SSMGR CV chart without the absorbing state. Let $T=1-P$ and $S=P$, then the $(g, h)$ th entry of matrix $\boldsymbol{R}$ is obtained as follows (Gadre et al ${ }^{19}$ ):

$$
R_{\mathrm{g}, h}=\left\{\begin{array}{c}
T \text { if the gth state leads to the } h \text { th state and the } h \text { th state corresponds to the } \\
\text { sequence ending with } 0 \text { (or } \tilde{0}) . \\
S \text { if the gth state leads to the } h \text { th state and the } h \text { th state corresponds to the } \\
\text { sequence ending with } G . \\
\alpha S \text { if the gth state leads to the } h \text { th state and the } h \text { th state corresponds to the } \\
\text { sequence ending with } \bar{G}(\text { or } \bar{H}) . \\
(1-\alpha) S \text { if the gth state leads to the } h \text { th state and the } h \text { th state corresponds } \\
\text { to the sequence ending with } \underline{G}(\text { or } \underline{H}) . \\
0 \text { otherwise. }
\end{array}\right.
$$

In general, the tpm $\boldsymbol{R}$ of the SSMGR CV chart is a square matrix with a dimension of $\left(3\left(C_{1}+C_{2}\right)+2\right) \times\left(3\left(C_{1}+C_{2}\right)+2\right)$. As an example, Table 3 presents the entries of $\boldsymbol{R}$ corresponding to the values $C_{1}=C_{2}=3$. The ARL and SDRL of the SSMGR CV chart, for a shift size $\tau$, are computed as 


$$
\operatorname{ARL}(\tau)=v_{1}
$$

and

$$
\operatorname{SDRL}(\tau)=\sqrt{v_{2}-v_{1}^{2}+v_{1}}
$$

respectively, where

$$
v_{1}=\boldsymbol{q}^{T}(\boldsymbol{I}-\boldsymbol{R})^{-1} \mathbf{1}
$$

and

$$
v_{2}=2 \boldsymbol{q}^{T}(\boldsymbol{I}-\boldsymbol{R})^{-2} \boldsymbol{R} \cdot \mathbf{1}
$$

Here, $\boldsymbol{q}^{T}=(1,0,0, \ldots, 0)$ is the initial probability vector and its dimension is $\left(3\left(C_{1}+C_{2}\right)+2\right) \times 1$,

$\boldsymbol{I}$ is the $\left(3\left(C_{1}+C_{2}\right)+2\right) \times\left(3\left(C_{1}+C_{2}\right)+2\right)$ identity matrix and $\mathbf{1}$ is a $\left(3\left(C_{1}+C_{2}\right)+2\right) 1$ column vector whose entries are all ones.

Let $\gamma_{1}$ be the value of the out-of-control CV and $\tau=\gamma_{1} / \gamma_{0}$ be the standardized CV shift from $\gamma_{0}$ to $\gamma_{1}$. By definition, an upward shift in the process $\mathrm{CV}$ occurs when $\tau>1$ and a downward shift in the process CV happens when $\tau<1$. The process $\mathrm{CV}$ is said to be in the in-control state when $\tau$ $=1$.

\section{Linear covariate error model for the $\mathrm{CV}$}

Let $\left\{X_{i, 1}, X_{i, 2}, \ldots, X_{i, n}\right\}$ denote the $i$ th sample of quality characteristics, where $X_{i, j} \sim$ $N\left(\mu_{0}+a \sigma_{0}, b^{2} \sigma_{0}^{2}\right)$, for $j=1,2, \ldots, n$ and $n>1$. Here, $\mu_{0}$ and $\sigma_{0}$ are the nominal mean and standard deviation, respectively. In addition, $a$ and $b$ denote the sizes of the standardized mean and standard deviation shifts, respectively. The process has shifted when $a \neq 0$ or/and $b \neq 1$. It is 
assumed that the quality characteristic $X_{i, j}$ cannot be observed directly but can only be obtained from the results $\left\{X_{i, j, 1}^{*}, X_{i, j, 2}^{*}, \ldots, X_{i, j, m}^{*}\right\}$ of a set of $m \geq 1$ characteristics, where each $X_{i, j, k}^{*}$ satisfies the following linear covariate error model (Tran et al ${ }^{38}$ ):

$$
X_{i, j, k}^{*}=A+B X_{i, j}+\varepsilon_{i, j, k} .
$$

Here, $A$ and $B$ are two known constants and $\varepsilon_{i, j, k}$ is a normal $N\left(0, \sigma_{M}^{2}\right)$ random error term due to measurement inaccuracy, which is supposed to be independent of $X_{i, j}$.

For sample $i(i=1,2, \ldots)$, there exists $m \times n$ observations $X_{i, j, k}^{*}$, for $j=1,2, \ldots, n$ and $k=1$, $2, \ldots, m$. Then, the mean $\bar{X}_{i, j}^{*}$ of the characteristics $\left\{X_{i, j, 1}^{*}, X_{i, j, 2}^{*}, \ldots, X_{i, j, m}^{*}\right\}$ is computed as (Tran et $\mathrm{al}^{38}$ )

$$
\begin{aligned}
\bar{X}_{i, j}^{*} & =\frac{1}{m} \sum_{k=1}^{m} X_{i, j, k}^{*} \\
& =\frac{1}{m} \sum_{k=1}^{m}\left(A+B X_{i, j}+\varepsilon_{i, j, k}\right) \\
& =A+B X_{i, j}+\frac{1}{m} \sum_{k=1}^{m} \varepsilon_{i, j, k} .
\end{aligned}
$$

The mean and standard deviation of $\bar{X}_{i, j}^{*}$ are (Tran et al ${ }^{38}$ )

$$
\mu^{*}=A+B\left(\mu_{0}+a \sigma_{0}\right)
$$

and

$$
\sigma^{*}=\sqrt{B^{2} b^{2} \sigma_{0}^{2}+\frac{\sigma_{M}^{2}}{m}}
$$

respectively. Consequently, the $\mathrm{CV}$ of the measured characteristic $\bar{X}_{i, j}^{*}$ is obtained as 


$$
\begin{aligned}
\gamma^{*}=\frac{\sigma^{*}}{\mu^{*}} & =\frac{\sqrt{B^{2} b^{2} \sigma_{0}^{2}+\sigma_{M}^{2} / m}}{A+B\left(\mu_{0}+a \sigma_{0}\right)} \\
& =\frac{\gamma_{0} \sqrt{B^{2} b^{2}+\eta^{2} / m}}{\theta+B\left(1+a \gamma_{0}\right)}
\end{aligned}
$$

where $\eta=\frac{\sigma_{M}}{\sigma_{0}}, \theta=\frac{A}{\mu_{0}}$ and $\gamma_{0}=\frac{\sigma_{0}}{\mu_{0}}$. Here, $\eta$ is called the precision error ratio, $\theta$ is the accuracy error and $\gamma_{0}$ is the nominal value of the population $\mathrm{CV}$. Note that $\gamma^{*}$ in Equation (18) no longer depends on parameter $A$.

Let $\overline{\bar{X}}_{i}^{*}$ and $S_{i}^{*}$ be the sample mean and sample standard deviation of $\left\{\bar{X}_{i, 1}^{*}, \bar{X}_{i, 2}^{*}, \ldots, \bar{X}_{i, n}^{*}\right\}$. Then (Tran et al ${ }^{38}$ )

$$
\overline{\bar{X}}_{i}^{*}=\frac{1}{n} \sum_{j=1}^{n} \bar{X}_{i, j}^{*}
$$

and

$$
S_{i}^{*}=\sqrt{\frac{1}{n-1} \sum_{j=1}^{n}\left(\bar{X}_{i, j}^{*}-\overline{\bar{X}}_{i}^{*}\right)^{2}}
$$

It follows that the $i$ th sample $\mathrm{CV}$ of the measured characteristic $\bar{X}_{i, j}^{*}$ is

$$
\hat{\gamma}_{i}^{*}=\frac{S_{i}^{*}}{\overline{\bar{X}}_{i}^{*}}
$$

The cdf and inverse cdf of $\hat{\gamma}^{*}$ can be obtained using Equations (5) and (6), respectively, by replacing $\gamma$ with $\gamma^{*}$ in Equation (18).

\section{SSMGR CV chart with measurement errors}


In the presence of measurement errors, the upper and lower control limits of the SSMGR CV-ME chart are denoted by $\mathrm{UCL}_{\mathrm{ME}}$ and $\mathrm{LCL}_{\mathrm{ME}}$, respectively. Both of these control limits can be computed using Equations (7a) and (7b) but by replacing $\hat{\gamma}, \gamma_{0}$ and $k$ with $\hat{\gamma}^{*}, \gamma_{0}^{*}$ and $k^{*}$, respectively. Note that the value of the $\mathrm{UCL}_{\mathrm{ME}}$ and $\mathrm{LCL}_{\mathrm{ME}}$ limits' constant $k^{*}$ is chosen to attain a desired in-control performance for the SSMGR CV-ME chart. The in-control CV of $\bar{X}_{i, j}^{*}$, i.e. $\gamma_{0}^{*}$ , required in the computation of $\mathrm{UCL}_{\mathrm{ME}}$ and $\mathrm{LCL}_{\mathrm{ME}}$ is calculated using Equation (18) by letting $a=0$ and $b=1$. The probability that the sample $\mathrm{CV}$ of $\bar{X}_{i, j}^{*}$ is non-conforming, denoted by $P^{*}$, is computed using Equation (8) but by substituting $\hat{\gamma}, \gamma, \mathrm{UCL}$ and LCL with $\hat{\gamma}^{*}, \gamma^{*}, \mathrm{UCL}_{\mathrm{ME}}$ and $\mathrm{LCL}_{\mathrm{ME}}$, respectively. The out-of-control $\gamma^{*}$, denoted as $\gamma_{1}^{*}$ is computed using Equation (18) when $b \neq 1$, where $1+a \gamma_{0}$ in Equation (18) can be replaced with $b / \tau$ so that a relation between $\gamma_{1}^{*}$ and $\tau$ exists (Tran et al ${ }^{38}$ ). It should be noted that $\gamma_{1}^{*}$ is used to compute $P^{*}$ when the process is outof-control.

The ARL and SDRL values of the SSMGR CV-ME chart are computed using Equations (10) and (11), respectively, by means of the Markov chain approach. Here, the computation of the tpm $\boldsymbol{R}$ is made in a similar way to that of the SSMGR CV chart discussed in Section 3, except that $P$ and $\alpha$ are replaced by $P^{*}$ and $\alpha^{*}$, respectively. Note that $\alpha^{*}$ is computed using Equation (9) but by replacing $\hat{\gamma}, P, \mathrm{UCL}$ and LCL with $\hat{\gamma}^{*}, P^{*}, \mathrm{UCL}_{\mathrm{ME}}$ and $\mathrm{LCL}_{\mathrm{ME}}$, respectively. The SSMGR CV-ME chart is implemented in the same way as that for the SSMGR CV chart (see the three step procedure) discussed in Section 3 but by replacing $\hat{\gamma}, \mathrm{UCL}$ and LCL with $\hat{\gamma}^{*}, \mathrm{UCL}_{\mathrm{ME}}$ and $\mathrm{LCL}_{\mathrm{ME}}$ , respectively. 


\section{Optimal designs}

The optimal designs of the SSMGR CV and SSMGR CV-ME charts involve the computation of the charts' respective optimal parameters to minimize $\operatorname{ARL}(\tau)$ for $\tau \neq 1$. For the SSMGR CV chart, the step-by-step procedure in computing the chart's optimal design parameters $k, C_{1}$ and $C_{2}$ is as follows:

Step 1. Specify the values of the desired in-control ARL (say ARL $(1)=\omega$ ), $\gamma_{0}$, shift size $\tau$ for which a quick detection is important and sample size $n$. Furthermore, initialize $C_{1}=0$, $C_{2}=0, \mathrm{ARL}_{\text {min }}=\infty$ and $\mathrm{ARL}_{\text {opt }}=\infty$

Step 2. Set $C_{1}=C_{1}+1$.

Step 3. Set $C_{2}=C_{2}+1$.

Step 4. Find the value of $k$ by numerically solving the limits UCL and LCL in Equations (7a) and (7b), respectively, so that $\operatorname{ARL}(1)=\omega$.

Step 5. Compute ARL $(\tau)$ using Equation (10) using the current values of $C_{1}, C_{2}$ and $k$.

Step 6. If $\operatorname{ARL}(\tau)<\mathrm{ARL}_{\text {min }}$, then let $\mathrm{ARL}_{\text {min }}=\operatorname{ARL}(\tau)$ and return to Step 3. Otherwise, proceed to Step 7.

Step 7. If $\mathrm{ARL}_{\text {min }}<\mathrm{ARL}_{\text {opt }}$, then let $\mathrm{ARL}_{\text {opt }}=\mathrm{ARL}_{\text {min }}$ and return to Step 2, while setting $C_{2}=0$. Otherwise, proceed to Step 8.

Step 8. The minimum value of $\operatorname{ARL}(\tau)$, based on the values of $\omega, \gamma_{0}, \tau$ and $n$ specified in Step 1 is given by $\mathrm{ARL}_{\text {opt }}$, while the corresponding values of $C_{1}, C_{2}$ and $k$ that produce this $\mathrm{ARL}_{\text {opt }}$ value are the optimal choices of parameters for the SSMGR CV chart. 
The above-mentioned step-by-step procedure can also be used to compute the optimal parameter combination $\left(C_{1}^{*}, C_{2}^{*}, k^{*}\right)$ of the SSMGR CV-ME chart. However, for this case, in addition to the values of $\omega, \gamma_{0}, \tau$ and $n$ specified in Step 1, the values of $\eta, B, m$ and $\theta$ also need to be specified in the same step. Furthermore, in the above eight step procedure, $C_{1}, C_{2}, k$, UCL and LCL are replaced by $C_{1}^{*}, C_{2}^{*}, k^{*}, \mathrm{UCL}_{\mathrm{ME}}$ and $\mathrm{LCL}_{\mathrm{ME}}$, respectively.

By adopting the above eight step procedure, optimization programs have been written in MATLAB to compute the optimal parameters of the SSMGR CV and SSMGR CV-ME charts that produce the minimum $\operatorname{ARL}(\tau)$ value (for $\tau \neq 1$ ), based on the desired input parameters specified in Step 1. The corresponding $\operatorname{SDRL}(\tau)$ values are also computed. Table 4 presents the optimal parameter combination $\left(C_{1}, C_{2}, k\right)$ that minimizes $\operatorname{ARL}(\tau)$ of the SSMGR CV chart for various choices of $n, \gamma_{0}$ and $\tau$. For example, when $\omega=370, n=5, \gamma_{0}=0.05$ and $\tau=0.5$ are considered, the optimal parameter combination of the SSMGR CV chart are $\left(k, C_{1}, C_{2}\right)=(0.0843,1,7)$, where these parameters produce the smallest value of $\operatorname{ARL}(0.25)(=3.12)$ (see Table 5) among all parameter combinations $\left(k, C_{1}, C_{2}\right)$ that give $\omega=370$. The SDRL $(0.25)$ value of the SSMGR CV chart corresponding to $\left(k, C_{1}, C_{2}\right)=(0.0843,1,7)$ is 4.25 (see Table 5).

Tables 6 - 9 present the optimal combinations of parameters $\left(k^{*}, C_{1}^{*}, C_{2}^{*}\right)$ for the SSMGR CV-ME chart that minimize $\operatorname{ARL}(\tau)$, as well as the corresponding $\operatorname{ARL}(\tau)$ and $\operatorname{SDRL}(\tau)$ values of the chart, for the specified $\gamma_{0}, \tau, n, \eta, B, m$ and $\theta$ values. Note that, without loss of generality, $b$ $=1$ is considered in this research. For example, when the SSMGR CV-ME chart is optimally designed to minimize $\operatorname{ARL}(0.5)$, i.e. for $\tau=0.5$, the optimal parameter combination $\left(k^{*}, C_{1}^{*}, C_{2}^{*}\right)$ 
$=(0.0843,1,7)$ is obtained with the corresponding $\operatorname{ARL}(0.5)=3.12$ and $\operatorname{SDRL}(0.5)=4.25$, when $n=5, \gamma_{0}=0.05, \eta=0.1, B=1, m=1$ and $\theta=0$ (see Table 6 ).

\section{Performance evaluation}

There are two objectives of this section. In Section 7.1, the performance of the proposed SSMGR $\mathrm{CV}$ chart is compared with those of existing CV charts, while in Section 7.2, the performance of the SSMGR CV-ME chart is investigated in the presence of measurement errors.

\subsection{Performance comparison of SSMGR CV and existing CV charts}

The performances of the proposed SSMGR CV and existing EWMA CV, RS CV and SSGR CV charts are compared using the $\operatorname{ARL}(\tau)$ and $\operatorname{SDRL}(\tau)$ criteria. The 7 regions $(7 \mathrm{R}) \mathrm{RS} \mathrm{CV}$ chart is considered as Teoh et al ${ }^{10}$ showed that the 7R RS CV chart is more efficient than its 4 regions counterpart. Additionally, the synthetic type CV charts are not considered in the comparison as You et al ${ }^{7}$ already showed that the SSGR CV chart outperforms the former.

In Table 5, it is obvious that the SSMGR CV chart outperforms the SSGR CV chart for all $\left(\gamma_{0}, \tau\right)$ combinations as the former has a smaller $\operatorname{ARL}(\tau)$ value than the latter for the same $\left(\gamma_{0}, \tau\right)$ combination. For example, when $\gamma_{0}=0.1$ and $\tau \in\{0.25,0.5,0.75,1.25,1.5,2\}, \operatorname{ARL}(\tau) \in\{1.01$, $3.14,52.57,8.89,3.13,1.53\}$ for the SSMGR CV chart, while that for the SSGR CV chart are $\{1.01,4.74,89.66,15.22,4.06,1.66\}$, where the $\operatorname{ARL}(\tau)$ values for the former are all lower than that of the latter. In comparison to the EWMA CV and RS CV charts, the SSMGR CV chart generally performs better for all $\tau$ values, except for $\tau=0.75$. For $\tau=0.75$, the EWMA CV and RS CV charts provide smaller ARL $(\tau)$ values compared to the SSMGR CV chart for all values of 
$\gamma_{0}$. For instance, when $\gamma_{0} \in\{0.05,0.1,0.15,0.2\}$, the ARL(0.75) values of the EWMA CV and RS CV charts are $\{17.19,17.30,17.49,17.78\}$ and $\{22.21,22.30,22.45,22.72\}$, respectively, while those of the SSMGR CV chart are $\{51.99,52.57,53.50,54.86\}$, where it is obvious that the ARL(0.75) values of the SSMGR CV chart are all greater than those of the EWMA CV and RS

CV charts. However, for values of $\tau \neq 0.75$, the SSMGR CV chart has smaller ARL $(\tau)$ values than those of the EWMA CV and RS CV charts (see Table 5).

In terms of the $\operatorname{SDRL}(\tau)$ criterion, it is also noticeable in Table 5 that the SSMGR CV chart (lower SDRL $(\tau)$ values) significantly prevails over the SSGR CV chart (higher SDRL( $\tau$ ) values), for all values of $\tau$ (except $\tau=0.75)$. In addition, for all $\gamma_{0}$ values based on the $\operatorname{SDRL}(\tau)$ criterion, the SSMGR CV chart is inferior to the EWMA CV chart when $0.5 \leq \tau \leq 1.25$, while the RS CV chart prevails over the SSMGR CV chart when $0.5 \leq \tau \leq 0.75$. For example, when $\gamma_{0}=0.1$ and $\tau$ $=0.5, \operatorname{SDRL}(0.5)$ of the EWMA CV, RS CV and SSMGR CV charts are 1.55, 1.51 and 4.30, respectively, where the values of $\operatorname{SDRL}(\tau)$ for the EWMA CV and RS CV charts are lower than those of the SSMGR CV chart. For the other values of $\tau$ not discussed above, the SSMGR CV chart outperforms the EWMA CV and RS CV charts based on the $\operatorname{SDRL}(\tau)$ criterion. For instance, when $\gamma_{0}=0.05$ and $\tau=0.25, \operatorname{SDRL}(0.25)=0.39,0.99$ and 0.10 for the EWMA CV, RS CV and SSMGR CV charts, respectively, where the SSMGR CV chart has the smallest SDRL(0.25) value.

\subsection{Performance of the SSMGR CV-ME chart in the presence of measurement errors}

Tables $6-9$ present the optimal combinations of parameters $\left(k^{*}, C_{1}^{*}, C_{2}^{*}\right)$, as well as the corresponding minimum $\operatorname{ARL}(\tau)$ values and $\operatorname{SDRL}(\tau)$ values of the SSMGR CV-ME chart based 
on the linear covariate error model, for fixed $\gamma_{0}, \tau, n, \eta, B, m$ and $\theta$ values that satisfy ARL(1) $=370$.

Table 6 shows the $\operatorname{ARL}(\tau)$ and $\operatorname{SDRL}(\tau)$ values of the SSMGR CV-ME chart for various combinations of the precision error ratios $\eta \in\{0,0.1,0.3,0.5,1\}, \gamma_{0} \in\{0.05,0.1,0.15,0.2\}$ and $\tau \in\{0.25,0.5,0.75,1.25,1.5,2\}$ when $n=5, m=1, B=1$ and $\theta=0$. Note that $\eta=0$ represents the case without measurement error. Additionally, note that when $\eta=\theta=0$ and $m=B=1$, the SSMGR CV-ME chart becomes the basic SSMGR CV chart (as in the case of Table 6). Thus, when $\eta=0$ in Table 6 , the optimal parameters $\left(k^{*}, C_{1}^{*}, C_{2}^{*}\right)$ that minimize $\operatorname{ARL}(\tau)$ of the $\operatorname{SSMGR}$ CV-ME chart are not given as they are actually similar to the optimal parameters $\left(k, C_{1}, C_{2}\right)$ of the SSMGR CV chart (without measurement error) in Table 4. It is obvious from Table 6 that the precision error ratio $(\eta)$ has a negative effect on the performance of the SSMGR CV-ME chart as, generally, there are slight increases in the $\operatorname{ARL}(\tau)$ and $\operatorname{SDRL}(\tau)$ values when $\eta$ increases. For example, when $\gamma_{0}=0.1$ and $\tau=0.75, \operatorname{ARL}(0.75) \in\{52.57,52.76,53.31\}$ and $\operatorname{SDRL}(0.75) \in$ $\{212.90,213.80,217.40\}$ for $\eta \in\{0,0.5,1\}$, where it is found that both $\operatorname{ARL}(0.75)$ and SDRL(0.75) increase slightly with $\eta$ (see Table 6). However, the results in Table 6 show that the precision error ratio $(\eta)$ does not have a significant effect on the performance of the SSMGR CVME chart.

Table 7 presents the $\operatorname{ARL}(\tau)$ and $\operatorname{SDRL}(\tau)$ performances of the SSMGR CV-ME chart for various combinations of $B \in\{1,2,3,4\}, \gamma_{0} \in\{0.05,0.1,0.15,0.2\}$ and $\tau \in\{0.25,0.5,0.75,1.25$, $1.5,2\}$ when $n=5, \eta=0.28, m=1$ and $\theta=0.01$. The rationale for choosing $\eta=0.28$ is due to the assumption of an acceptable value for the signal-to-noise ratio as explained in Tran at al. ${ }^{33}$ In 
Table 7 , it is noticeable that for the fixed values of $n, \gamma_{0}, \tau, \eta, m$ and $\theta$, the value of $B$ has some positive effects on the performance of the SSMGR CV-ME chart as, generally, the ARL $(\tau)$ and $\operatorname{SDRL}(\tau)$ values decrease slightly when $B$ increases. As an example, when $\gamma_{0}=0.15, \tau=0.75$, $\operatorname{ARL}(0.75)=55.14$ and $\operatorname{SDRL}(0.75)=225.91$ for $B=1$, while $\operatorname{ARL}(0.75)=53.88$ and $\operatorname{SDRL}(0.75)$ $=220.08$ for $B=4$ (see Table 7), i.e. both ARL(0.75) and SDRL(0.75) decrease when $B$ increases from 1 to 5 .

Linna and Woodall ${ }^{27}$ noted that it is better to take multiple measurements per item in each sample to reduce the effect of measurement errors. Table 8 presents the $\operatorname{ARL}(\tau)$ and $\operatorname{SDRL}(\tau)$ values of the SSMGR CV-ME chart for various combinations of $m \in\{1,3,5,7\}, \gamma_{0} \in\{0.05,0.1$, $0.15,0.2\}$ and $\tau \in\{0.25,0.5,0.75,1.25,1.5,2\}$ when $n=5, \eta=0.28, B=1$ and $\theta=0.01$. Table 8 shows that increasing the number of measurements per item $(m)$ gives some positive effect on the SSMGR CV-ME chart by slightly reducing the $\operatorname{ARL}(\tau)$ and $\operatorname{SDRL}(\tau)$ values of the chart when $\gamma_{0}, \tau, n, \eta, B$ and $\theta$ are fixed, though the reduction is not large. For example, when $\gamma_{0}=0.2$ and $\tau$ $=0.75, \operatorname{ARL}(0.75)=56.62$ and $\operatorname{SDRL}(0.75)=232.77$ for $m=1$, while $\operatorname{ARL}(0.75)=56.41$ and $\operatorname{SDRL}(0.75)=231.80$ for $m=7$ (see Table 8 ). It is obvious that the values of ARL $(0.75)$ and SDRL(0.75) reduce when $m$ increases from 1 to 7.

Table 9 shows that the $\operatorname{ARL}(\tau)$ and $\operatorname{SDRL}(\tau)$ values of the SSMGR CV-ME chart are slightly negatively influenced by the accuracy error $(\theta)$ when $\gamma_{0}, \tau, n(=5), \eta(=0.28), B(=1)$ and $m(=1)$ are fixed. For instance, when $\gamma_{0}=0.2$ and $\tau=1.25, \operatorname{ARL}(1.25)=9.31$ and $\operatorname{SDRL}(1.25)=17.70$ for $\theta=0$, while $\operatorname{ARL}(1.25)=10.24$ and $\operatorname{SDRL}(1.25)=20.30$ for $\theta=0.05$ (see Table 9), where the values of ARL(1.25) and SDRL(1.25) increase with $\theta$. 


\section{Implementations}

To illustrate the implementation of the SSMGR CV and SSMGR CV-ME charts, real life data from a Tunisian company that manufactures sanitary parts from zinc alloy in a die casting hot chamber process are adopted from Castagliola et al ${ }^{4}$ The quality characteristic of interest $X$ is the weight (in grams) of scrap zinc alloy material (see Castagliola et al ${ }^{4}$ for details of the process). Table 10 gives the Phase-I and Phase-II datasets of this quality characteristic. A regression study was conducted by Castagliola et $\mathrm{al}^{4}$ on the 30 Phase-I samples, each having 5 observations, where a constant proportionality $(\sigma=\gamma \times \mu)$ between the process standard deviation $\sigma$ and the process mean $\mu$ of the weight of scrap zinc alloy was found to exist. Castagliola et al ${ }^{4}$ also showed that the Phase-I data are in-control. By adopting the root mean square method on the Phase-I samples, the estimated in-control $\mathrm{CV}\left(\hat{\gamma}_{0}\right)$ is computed as

$$
\hat{\gamma}_{0}=\sqrt{\frac{1}{30} \sum_{i=1}^{30} \hat{\gamma}_{i}^{2}}=0.0108,
$$

which can be rounded to $\hat{\gamma}_{0}=0.01$.

Suppose that a process engineer has decided to implement the SSMGR CV and SSMGR CVME charts for monitoring the Phase-II process. It is assumed that the SSMGR CV chart is optimally designed, based on $\operatorname{ARL}(1)=370, n=5$ and $\gamma_{0}=0.01$ (as $\hat{\gamma}_{0} \approx 0.01$ ); while the SSMGR CV-ME chart is optimally designed based on $\operatorname{ARL}(1)=370, n=5, \gamma_{0}=0.01, \eta=0.28, B=1, m$ $=1$ and $\theta=0$. An upward shift in the process $\mathrm{CV}$, where a quick detection is important is set as $\tau$ $=1.5$, for both the charts. Consequently, the optimal parameters $k=0.0701, C_{1}=1$ and $C_{2}=11$ are computed using the Matlab optimization program for the SSMGR CV chart, where the limits of the chart are computed to be $\mathrm{UCL}=0.0161$ and $\mathrm{LCL}=0.0038$. Similarly, the optimal parameters 
of the SSMGR CV-ME chart, i.e. $k^{*}=0.0701, C_{1}^{*}=1$ and $C_{2}^{*}=11$, are computed, which result in $\mathrm{UCL}_{\mathrm{ME}}=0.0167$ and $\mathrm{LCL}_{\mathrm{ME}}=0.0040$

From the control limits of the two charts given in the previous paragraph, it is found that the SSMGR CV chart has tighter limits (narrower width between UCL and LCL, where UCL - LCL $=0.0123$ ) than the SSMGR CV-ME chart (wider width between $\mathrm{UCL}_{\mathrm{ME}}$ and $\mathrm{LCL}_{\mathrm{ME}}$, where $\left.\mathrm{UCL}_{\mathrm{ME}}-\mathrm{LCL}_{\mathrm{ME}}=0.0127\right)$. Therefore, in general, it becomes easier for the SSMGR CV chart to issue an out-of-control signal, either due to the occurrence of an assignable cause or the presence of measurement errors or both, as the chart's limits have become tighter. However, for the SSMGR CV-ME chart, as its limits have become looser (compared to the SSMGR CV chart), it is in general more difficult to detect an out-of-control signal (compared to the SSMGR CV chart). Hence, when the SSMGR CV-ME chart detects an out-of-control signal, the signal is more likely due to actual process shifts, instead of measurement errors, as its limits have been computed by considering the presence of measurement errors.

The 30 Phase-II sample CVs $\left(\hat{\gamma}_{i}\right.$, for $\left.i=1,2, \ldots, 30\right)$ are plotted in Figure 1. From Figure 1, it is obvious that the $1^{\text {st }}$ non-conforming $\hat{\gamma}_{i}$ occurs at sample no., $i=9$, for both the SSMGR CV and SSMGR CV-ME charts. Thus, $\mathrm{CRL}_{1}=9$ is obtained. The $2^{\text {nd }}, 3^{\text {rd }}$ and $4^{\text {th }}$ non-conforming samples are observed at sample nos., $i=10,12$ and 13, hence, $\mathrm{CRL}_{2}=1, \mathrm{CRL}_{3}=2$ and $\mathrm{CRL}_{4}=1$ are computed for both the charts. Since $\mathrm{CRL}_{1}(=9) \leq C_{2}=C_{2}^{*}(=11)$, then according to Step 3 of the implementation procedure in Section 3, the SSMGR CV and SSMGR CV-ME charts issue the first out-of-control signal at sample $9(i=9)$ (see Figure 1). Following this out-of-control signal, corrective actions should be taken so that the out-of-control process returns to the in-control situation again. 


\section{Conclusions}

There are many circumstances where the mean and standard deviation of a manufacturing process vary in a proportional manner. The $\mathrm{CV}$ is a suitable quality characteristic for monitoring the process stability of this type of process. In this research, we have introduced a new CV chart, called the SSMGR CV chart, for efficiently monitoring the CV. The SSMGR CV chart has been compared with the existing EWMA CV, RS CV and SSGR CV charts using the ARL and SDRL as performance criteria. The ARL results have shown that the SSMGR CV chart outperforms the three aforementioned existing $\mathrm{CV}$ charts in detecting increasing $\mathrm{CV}$ shifts $(\tau>1)$; while for detecting decreasing CV shifts $(\tau<1)$, the SSMGR CV-ME chart still prevails, except for $\tau=0.75$, where the EWMA CV and RS CV charts beat the former. Based on the findings in this research, we recommend the use of the SSMGR CV chart when the process engineer is interested to monitor upward shifts $(\tau>1)$ or moderate-to-large downward shifts $(\tau<1)$, in the process CV.

When a process being monitored is in-control, ideally the ratio of the standard deviation to the mean, i.e. $\gamma_{0}=\sigma_{0} / \mu_{0}$ should be small so that the process is operating reliably. Note that the data employed to illustrate the implementations of the $\mathrm{CV}$ charts in practice by Kang et al, ${ }^{1} \mathrm{Khaw}$ et al, ${ }^{11}$ and Yeong et al, ${ }^{42}$ all deal with small $\gamma_{0}$ values, namely $0.075,0.005645$ and 0.001042 , respectively. Thus, small $\gamma_{0}$ values, i.e. $\gamma_{0} \in\{0.05,0.1,0.15,0.2\}$ are adopted in this article.

This research has also investigated the effect of measurement errors on the SSMGR CV (referred to as the SSMGR CV-ME) chart. The findings has revealed that the increases in the precision error ratio $(\eta)$ or accuracy error $(\theta)$ has a negative effect on the SSMGR CV-ME chart's performance by slightly increasing its $\operatorname{ARL}(\tau)$ and $\operatorname{SDRL}(\tau)$ values for a similar shift size $\tau$. Furthermore, increasing the number of measurements per item $(m)$ or the parameter $B$ has a 
positive effect on the $\operatorname{ARL}(\tau)$ and $\operatorname{SDRL}(\tau)$ performances of the SSMGR CV-ME chart as their values decrease slightly when $m$ or $B$ increases.

In this research, the univariate SSMGR CV and SSMGR CV-ME charts have been developed to monitor the process $\mathrm{CV}$. In the future, research may be conducted to develop the multivariate SSMGR CV and SSMGR CV-ME charts. Moreover, univariate and multivariate SSMGR CV and SSMGR CV-ME charts with estimated process parameters, i.e. when the target values of the process parameters are unknown or cannot be specified, can also be proposed.

\section{Acknowledgments}

This work is supported by the Miyan Research Institute, International University of Business Agriculture and Technology, Dhaka, Bangladesh.

\section{References}

1. Kang C, Lee M, Seong Y, Hawkins D. A control chart for the coefficient of variation. Journal of Quality Technology 2007; 39: 151-158.

2. Hong EP, Kang CW, Baek JW, Kang HW. Development of CV control chart using EWMA technique. Journal of the Society of Korea Industrial and Systems Engineering 2008; 31: $114-$ 120.

3. Castagliola P, Celano G, Psarakis S. Monitoring the coefficient of variation using EWMA charts. Journal of Quality Technology 2011; 43: 249-265.

4. Castagliola P, Achouri A, Taleb, H, Celano G, Psarakis S. Monitoring the coefficient of variation using a variable sampling interval control chart. Quality and Reliability Engineering International 2013; 29: 1135-1149. 
5. Castagliola P, Achouri A, Taleb H, Celano G, Psarakis S. Monitoring the coefficient of variation using a variable sample size control chart. The International Journal of Advanced Manufacturing Technology 2015; 80: 1561-1576.

6. Calzada ME, Scariano SM. A synthetic control chart for the coefficient of variation. Journal of Statistical Computation and Simulation 2013; 83: 853-867.

7. You HW, Khoo MBC, Castagliola P, Haq A. Monitoring the coefficient of variation using the side sensitive group runs chart. Quality and Reliability Engineering International 2016; 32: 1913-1927.

8. Noor-ul-Amin M, Tariq S, Hanif M. Control charts for simultaneously monitoring of process mean and coefficient of variation with and without auxiliary information. Quality and Reliability Engineering International 2019; 35: 2639-2656.

9. Giner-Bosch V, Tran KP, Castagliola P, Khoo MBC. An EWMA control chart for the multivariate coefficient of variation. Quality and Reliability Engineering International 2019; 35: 1515-1541.

10. Teoh WL, Khoo MBC, Castagliola P, Yeong WC, Teh SY. Run-sum control charts for monitoring the coefficient of variation. European Journal of Operational Research 2017; 257: 144-158.

11. Khaw KW, Khoo MBC, Yeong WC, Wu Z. Monitoring the coefficient of variation using a variable sample size and sampling interval control chart. Communications in StatisticsSimulation and Computation 2017; 46: 5772-5794.

12. Yeong WC, Khoo MBC, Lim SL, Teoh WL. The coefficient of variation chart with measurement error. Quality Technology \& Quantitative Management 2017; 14: 353-377. 
13. Yeong WC, Lim SL, Khoo MBC, Castagliola P. Monitoring the coefficient of variation using a variable parameters chart. Quality Engineering 2018; 30: 212-235.

14. Chew XY, Khoo MBC, Khaw KW, Yeong WC, Chong ZL. A proposed variable parameter control chart for monitoring the multivariate coefficient of variation. Quality and Reliability Engineering International 2019; 35: 2442-2461.

15. Wu Z, Spedding TA. A synthetic control chart for detecting small shifts in the process mean. Journal of Quality Technology 2000; 32: 32-38.

16. Gadre MP, Rattihalli RN. A group runs control chart for detecting shifts in the process mean. Economic Quality Control 2004; 19: 29-43.

17. Gadre MP, Rattihalli RN. Modified group runs control charts to detect increases in fraction nonconforming and shifts in the process mean. Communications in Statistics - Simulation and Computation 2006; 35: 225-240.

18. Gadre MP, Rattihalli RN. A side sensitive group runs control chart for detecting shifts in the process mean. Statistical Methods and Applications 2007; 16: 27-37.

19. Gadre MP, Joshi KA, Rattihalli RN. A side sensitive modified group runs control chart to detect shifts in the process mean. Journal of Applied Statistics 2010; 37: 2073-2087.

20. You HW, Khoo MBC, Castagliola P, Ou Y. Side sensitive group runs $\bar{X}$ chart with estimated process parameters. Computational Statistics 2015; 30: 1245-1278.

21. Khoo MBC, Tan EK, Chong ZL, Haridy S. Side-sensitive group runs double sampling (SSGRDS) chart for detecting mean shifts. International Journal of Production Research 2015; 53: 4735-4753.

22. Chong ZL, Khoo MBC, Lee $\mathrm{MH}$, Chen $\mathrm{CH}$. Group runs revised m-of-k runs rule control chart. Communications in Statistics - Theory and Methods 2017; 46: 6916-6935. 
23. Saha S, Khoo MBC, Lee MH, Castagliola P. A side-sensitive modified group runs double sampling (SSMGRDS) control chart for detecting mean shifts. Communications in Statistics - Simulation and Computation 2018; 47: 1353-1369.

24. Chong ZL, Khoo MBC, Teoh WL, You HW, Castagliola P. Optimal design of the sidesensitive modified group runs (SSMGR) chart when process parameters are estimated. Quality and Reliability Engineering International 2019; 35: 246-262.

25. Mim FN, Saha S, Khoo MBC, Khatun M. A Side-sensitive modified group runs control chart with auxiliary information to detect process mean shifts. Pertanika Journal of Science \& Technology 2019; 27: $847-866$.

26. Gadre MP, Kakade VC. Some side sensitive group runs based control charts to detect shifts in the process median. Communications in Statistics - Simulation and Computation 2019; doi.org/10.1080/03610918.2019.1672736.

27. Linna KW, Woodall WH. Effect of measurement error on Shewhart control charts. Journal of Quality Technology 2001; 33: 213-222.

28. Linna KW, Woodall WH, Busby KL. The performance of multivariate control charts in the presence of measurement error. Journal of Quality Technology 2001; 33; 349-355.

29. Costa AFB, Castagliola P. Effect of measurement error and autocorrelation on the $\bar{X}$ chart. Journal of Applied Statistics 2011; 38: 661-673.

30. Nguyen HD, Tran KP. Effect of the measurement errors on two one-sided Shewhart control charts for monitoring the ratio of two normal variables. Quality and Reliability Engineering International 2020: doi.org/10.1002/qre.2656. 
31. Tran PH, Tran KP, Rakitzis A. A Synthetic median control chart for monitoring the process mean with measurement errors. Quality and Reliability Engineering International 2019; 35: $1100-1116$.

32. Maravelakis PE. Measurement error effect on the CUSUM control chart. Journal of Applied Statistics 2012; 39: 323-336.

33. Hu X, Castagliola P, Sun J, Khoo MBC. The effect of measurement errors on the synthetic chart. Quality and Reliability Engineering International 2015; 31: 1769-1778.

34. Noorossana R, Zerehsaz Y. Effect of measurement error on phase II monitoring of simple linear profiles. The International Journal of Advanced Manufacturing Technology 2015; 79: 2031-2040.

35. Tran KP, Castagliola P, Celano G. The performance of the Shewhart-RZ control chart in the presence of measurement error. International Journal of Production Research 2016; 54: $7504-7522$.

36. Tran KP, Castagliola P, Balakrishnan N. On the performance of Shewhart median chart in the presence of measurement errors. Quality and Reliability Engineering International 2017; 33: 1019-1029.

37. Yeong WC, Khoo MBC, Tham LK, Teoh WL, Rahim MA. Monitoring the coefficient of variation using a variable sampling interval EWMA chart. Journal of Quality Technology 2017; 49: 380-401.

38. Tran KP, Heuchenne C, Balakrishnan N. On the performance of coefficient of variation charts in the presence of measurement errors. Quality and Reliability Engineering International 2019; 35: 329-350. 
39. McKay AT. Distribution of the Coefficient of Variation and the Extended " $t$ " Distribution. Journal of the Royal Statistical Society 1932; 95: 695-698.

40. Iglewicz B, Myers RH, Howe RB. On the percentage points of the sample coefficient of variation. Biometrika 1968; 55: 580-581.

41. Iglewicz B, Myers RH. Comparisons of Approximations to the Percentage Points of the Sample Coefficient of Variation. Technometrics 1970; 12: 166-169.

42. Yeong WC, Khoo MBC, Teoh WL, Castagliola P. A control chart for the multivariate coefficient of variation. Quality and Reliability Engineering International 2016; 32: 12131225. 
Table 1. Descriptions of the Markov chain states of the samples for the SSMGR CV chart

\begin{tabular}{|c|c|c|}
\hline State & Description & Description \\
\hline$\overline{\bar{G}}$ & $\begin{array}{l}\text { The sample at time zero is non- } \bar{G}(\bar{H}) \\
\text { conforming and the corresponding } \\
\text { CRL does not exceed } C_{1} \text {, where } \\
\text { there is either an upward or a } \\
\text { downward process mean shift. }\end{array}$ & $\begin{array}{l}\text { A sequence showing the non- } \\
\text { absorbing state, ending with the } \\
\text { sample in the first (second) level of } \\
\text { sample inspection being non- } \\
\text { conforming with an upward process } \\
\text { mean shift. }\end{array}$ \\
\hline$G$ & $\begin{array}{l}\text { The non-conforming sample in the } \underline{G}(\underline{H}) \\
\text { first level of sample inspection has a } \\
\text { process mean shift on any side with } \\
\text { CRL }>C_{1} .\end{array}$ & $\begin{array}{l}\text { A sequence showing the non- } \\
\text { absorbing state, ending with the } \\
\text { sample in the first (second) level of } \\
\text { sample inspection being non- } \\
\text { conforming with a downward process } \\
\text { mean shift. }\end{array}$ \\
\hline $0(\tilde{0})$ & $\begin{array}{l}\text { The sample in the first (second) } \\
\text { level of sample inspection is } \\
\text { conforming. }\end{array}$ & \\
\hline
\end{tabular}


Table 2. The Markov chain states of the SSMGR CV chart for $C_{1}=C_{2}=3$

\begin{tabular}{cccccc}
\hline State no. & State & State no. & State & State no. & State \\
\hline 1 & $\overline{\bar{G}}$ & 8 & $\bar{G} \tilde{0}$ & 15 & $\bar{H}$ \\
2 & $\overline{\bar{G}} \tilde{0}$ & 9 & $\bar{G} \tilde{0} \tilde{0}$ & 16 & $\bar{H} 0$ \\
3 & $\overline{\bar{G}} \tilde{0} \tilde{0}$ & 10 & $\underline{G} \tilde{0}$ & 17 & $\bar{H} 00$ \\
4 & $\tilde{0} \tilde{0} \tilde{0}$ & 11 & $\underline{G} \tilde{0} \tilde{0}$ & 18 & $\underline{H}$ \\
5 & $\bar{G}$ & 12 & $G$ & 19 & $\underline{H} 0$ \\
6 & $\underline{G}$ & 13 & $G 0$ & 20 & $\underline{H} 00$ \\
7 & 000 & 14 & $G 00$ & 21 & Signal \\
\hline
\end{tabular}


Table 3. The tpm of the Markov chain model of the SSMGR CV chart for $C_{1}=C_{2}=3$

\begin{tabular}{|c|c|c|c|c|c|c|c|c|c|c|c|c|c|c|c|c|c|c|c|c|}
\hline \multirow{2}{*}{$\begin{array}{c}\text { State } \\
g\end{array}$} & \multicolumn{20}{|c|}{ State $h$} \\
\hline & 1 & 2 & 3 & 4 & 5 & 6 & 7 & 8 & 9 & 10 & 11 & 12 & 13 & 14 & 15 & 16 & 17 & 18 & 19 & 20 \\
\hline 1 & 0 & $T$ & 0 & 0 & 0 & 0 & 0 & 0 & 0 & 0 & 0 & 0 & 0 & 0 & 0 & 0 & 0 & 0 & 0 & 0 \\
\hline 2 & 0 & 0 & $T$ & 0 & 0 & 0 & 0 & 0 & 0 & 0 & 0 & 0 & 0 & 0 & 0 & 0 & 0 & 0 & 0 & 0 \\
\hline 3 & 0 & 0 & 0 & $T$ & 0 & 0 & 0 & 0 & 0 & 0 & 0 & 0 & 0 & 0 & 0 & 0 & 0 & 0 & 0 & 0 \\
\hline 4 & 0 & 0 & 0 & $T$ & 0 & 0 & 0 & 0 & 0 & 0 & 0 & $S$ & 0 & 0 & 0 & 0 & 0 & 0 & 0 & 0 \\
\hline 5 & 0 & 0 & 0 & 0 & 0 & 0 & 0 & $T$ & 0 & 0 & 0 & 0 & 0 & 0 & 0 & 0 & 0 & $(1-\alpha) S$ & 0 & 0 \\
\hline 6 & 0 & 0 & 0 & 0 & 0 & 0 & 0 & 0 & 0 & $T$ & 0 & 0 & 0 & 0 & $\alpha S$ & 0 & 0 & 0 & 0 & 0 \\
\hline 7 & 0 & 0 & 0 & 0 & 0 & 0 & $T$ & 0 & 0 & 0 & 0 & $S$ & 0 & 0 & 0 & 0 & 0 & 0 & 0 & 0 \\
\hline 8 & 0 & 0 & 0 & 0 & 0 & 0 & 0 & 0 & $T$ & 0 & 0 & 0 & 0 & 0 & 0 & 0 & 0 & $(1-\alpha) S$ & 0 & 0 \\
\hline 9 & 0 & 0 & 0 & $T$ & 0 & 0 & 0 & 0 & 0 & 0 & 0 & 0 & 0 & 0 & 0 & 0 & 0 & $(1-\alpha) S$ & 0 & 0 \\
\hline 10 & 0 & 0 & 0 & 0 & 0 & 0 & 0 & 0 & 0 & 0 & $T$ & 0 & 0 & 0 & $\alpha S$ & 0 & 0 & 0 & 0 & 0 \\
\hline 11 & 0 & 0 & 0 & $T$ & 0 & 0 & 0 & 0 & 0 & 0 & 0 & 0 & 0 & 0 & $\alpha S$ & 0 & 0 & 0 & 0 & 0 \\
\hline 12 & 0 & 0 & 0 & 0 & $\alpha S$ & $(1-\alpha) S$ & 0 & 0 & 0 & 0 & 0 & 0 & $T$ & 0 & 0 & 0 & 0 & 0 & 0 & 0 \\
\hline 13 & 0 & 0 & 0 & 0 & $\alpha S$ & $(1-\alpha) S$ & 0 & 0 & 0 & 0 & 0 & 0 & 0 & $T$ & 0 & 0 & 0 & 0 & 0 & 0 \\
\hline 14 & 0 & 0 & 0 & 0 & $\alpha S$ & $(1-\alpha) S$ & $T$ & 0 & 0 & 0 & 0 & 0 & 0 & 0 & 0 & 0 & 0 & 0 & 0 & 0 \\
\hline 15 & 0 & 0 & 0 & 0 & $\alpha S$ & $(1-\alpha) S$ & 0 & 0 & 0 & 0 & 0 & 0 & 0 & 0 & 0 & $T$ & 0 & 0 & 0 & 0 \\
\hline 16 & 0 & 0 & 0 & 0 & $\alpha S$ & $(1-\alpha) S$ & 0 & 0 & 0 & 0 & 0 & 0 & 0 & 0 & 0 & 0 & $T$ & 0 & 0 & 0 \\
\hline 17 & 0 & 0 & 0 & 0 & $\alpha S$ & $(1-\alpha) S$ & $T$ & 0 & 0 & 0 & 0 & 0 & 0 & 0 & 0 & 0 & 0 & 0 & 0 & 0 \\
\hline 18 & 0 & 0 & 0 & 0 & $\alpha S$ & $(1-\alpha) S$ & 0 & 0 & 0 & 0 & 0 & 0 & 0 & 0 & 0 & 0 & 0 & 0 & $T$ & 0 \\
\hline 19 & 0 & 0 & 0 & 0 & $\alpha S$ & $(1-\alpha) S$ & 0 & 0 & 0 & 0 & 0 & 0 & 0 & 0 & 0 & 0 & 0 & 0 & 0 & $T$ \\
\hline 20 & 0 & 0 & 0 & 0 & $\alpha S$ & $(1-\alpha) S$ & $T$ & 0 & 0 & 0 & 0 & 0 & 0 & 0 & 0 & 0 & 0 & 0 & 0 & 0 \\
\hline
\end{tabular}


Table 4. Optimal parameters of the SSMGR CV chart when $\omega=370$

\begin{tabular}{|c|c|c|c|c|c|c|c|c|c|c|}
\hline \multirow[b]{2}{*}{$\gamma_{0}$} & \multirow[b]{2}{*}{$\tau$} & \multicolumn{3}{|c|}{$n=5$} & \multicolumn{3}{|c|}{$n=7$} & \multicolumn{3}{|c|}{$n=10$} \\
\hline & & $k$ & $C_{1}$ & $C_{2}$ & $k$ & $C_{1}$ & $C_{2}$ & $k$ & $C_{1}$ & $C_{2}$ \\
\hline \multirow[t]{6}{*}{0.05} & 0.25 & 0.1359 & 1 & 2 & 0.1359 & 1 & 2 & 0.1359 & 1 & 2 \\
\hline & 0.5 & 0.0843 & 1 & 7 & 0.1169 & 1 & 3 & 0.1359 & 1 & 2 \\
\hline & 0.75 & 0.0254 & 1 & 92 & 0.0365 & 1 & 46 & 0.0530 & 1 & 21 \\
\hline & 1.25 & 0.0430 & 1 & 33 & 0.0499 & 1 & 24 & 0.0582 & 1 & 17 \\
\hline & 1.5 & 0.0701 & 1 & 11 & 0.0799 & 1 & 8 & 0.0896 & 1 & 6 \\
\hline & 2 & 0.0962 & 1 & 5 & 0.1049 & 1 & 4 & 0.1169 & 1 & 3 \\
\hline \multirow[t]{6}{*}{0.1} & 0.25 & 0.1359 & 1 & 2 & 0.1359 & 1 & 2 & 0.1359 & 1 & 2 \\
\hline & 0.5 & 0.0843 & 1 & 7 & 0.1169 & 1 & 3 & 0.1359 & 1 & 2 \\
\hline & 0.75 & 0.0254 & 1 & 92 & 0.0361 & 1 & 47 & 0.0530 & 1 & 21 \\
\hline & 1.25 & 0.0430 & 1 & 33 & 0.0499 & 1 & 24 & 0.0582 & 1 & 17 \\
\hline & 1.5 & 0.0701 & 1 & 11 & 0.0799 & 1 & 8 & 0.0896 & 1 & 6 \\
\hline & 2 & 0.0962 & 1 & 5 & 0.1049 & 1 & 4 & 0.1169 & 1 & 3 \\
\hline \multirow[t]{6}{*}{0.15} & 0.25 & 0.1359 & 1 & 2 & 0.1359 & 1 & 2 & 0.1359 & 1 & 2 \\
\hline & 0.5 & 0.0843 & 1 & 7 & 0.1049 & 1 & 4 & 0.1359 & 1 & 2 \\
\hline & 0.75 & 0.0251 & 1 & 94 & 0.0357 & 1 & 48 & 0.0519 & 1 & 22 \\
\hline & 1.25 & 0.0424 & 1 & 34 & 0.0489 & 1 & 25 & 0.0582 & 1 & 17 \\
\hline & 1.5 & 0.0701 & 1 & 11 & 0.0799 & 1 & 8 & 0.0896 & 1 & 6 \\
\hline & 2 & 0.0962 & 1 & 5 & 0.1049 & 1 & 4 & 0.1169 & 1 & 3 \\
\hline \multirow[t]{6}{*}{0.2} & 0.25 & 0.1359 & 1 & 2 & 0.1359 & 1 & 2 & 0.1359 & 1 & 2 \\
\hline & 0.5 & 0.0843 & 1 & 7 & 0.1049 & 1 & 4 & 0.1359 & 1 & 2 \\
\hline & 0.75 & 0.0251 & 1 & 94 & 0.0350 & 1 & 50 & 0.0508 & 1 & 23 \\
\hline & 1.25 & 0.0418 & 1 & 35 & 0.0489 & 1 & 25 & 0.0567 & 1 & 18 \\
\hline & 1.5 & 0.0701 & 1 & 11 & 0.0799 & 1 & 8 & 0.0896 & 1 & 6 \\
\hline & 2 & 0.0962 & 1 & 5 & 0.1049 & 1 & 4 & 0.1169 & 1 & 3 \\
\hline
\end{tabular}


Table 5. ARL $(\tau)$ and SDRL $(\tau)$ values of the EWMA CV, RS CV, SSGR CV and SSMGR CV charts when $\omega=370$ and $n=5$

\begin{tabular}{|c|c|c|c|c|c|c|c|c|c|}
\hline \multirow[b]{2}{*}{$\gamma_{0}$} & \multirow[b]{2}{*}{$\tau$} & \multicolumn{2}{|c|}{ EWMA CV } & \multicolumn{2}{|c|}{ RS CV } & \multicolumn{2}{|c|}{ SSGR CV } & \multicolumn{2}{|c|}{ SSMGR CV } \\
\hline & & $\operatorname{ARL}(\tau)$ & $\operatorname{SDRL}(\tau)$ & $\operatorname{ARL}(\tau)$ & $\operatorname{SDRL}(\tau)$ & $\operatorname{ARL}(\tau)$ & $\operatorname{SDRL}(\tau)$ & $\operatorname{ARL}(\tau)$ & $\operatorname{SDRL}(\tau)$ \\
\hline$\overline{0.05}$ & 0.25 & 3.10 & 0.39 & 2.72 & 0.99 & 1.01 & 0.19 & 1.01 & 0.10 \\
\hline & 0.5 & 5.92 & 1.54 & 5.42 & 1.50 & 4.70 & 6.62 & 3.12 & 4.25 \\
\hline & 0.75 & 17.19 & 7.03 & 22.21 & 17.12 & 88.90 & 122.69 & 51.99 & 210.20 \\
\hline & 1.25 & 15.58 & 11.38 & 18.92 & 16.96 & 15.02 & 22.02 & 8.79 & 16.22 \\
\hline & 1.5 & 5.64 & 4.00 & 5.94 & 4.53 & 4.00 & 4.68 & 3.09 & 3.42 \\
\hline & 2 & 2.32 & 1.51 & 2.34 & 1.51 & 1.64 & 1.28 & 1.52 & 1.00 \\
\hline 0.1 & 0.25 & 3.13 & 0.38 & 2.78 & 1.04 & 1.01 & 0.19 & 1.01 & 0.10 \\
\hline & 0.5 & 5.99 & 1.55 & 5.44 & 1.51 & 4.74 & 6.69 & 3.14 & 4.30 \\
\hline & 0.75 & 17.30 & 7.04 & 22.30 & 17.19 & 89.66 & 123.67 & 52.57 & 212.90 \\
\hline & 1.25 & 15.64 & 11.44 & 19.09 & 17.16 & 15.22 & 22.37 & 8.89 & 16.60 \\
\hline & 1.5 & 5.69 & 4.02 & 6.01 & 4.60 & 4.06 & 4.78 & 3.13 & 3.50 \\
\hline & 2 & 2.35 & 1.53 & 2.38 & 1.54 & 1.66 & 1.32 & 1.53 & 1.03 \\
\hline 0.15 & 0.25 & 3.19 & 0.41 & 2.89 & 1.13 & 1.01 & 0.20 & 1.01 & 0.10 \\
\hline & 0.5 & 6.09 & 1.54 & 5.46 & 1.53 & 4.81 & 6.80 & 3.18 & 4.39 \\
\hline & 0.75 & 17.49 & 7.17 & 22.45 & 17.33 & 90.93 & 125.31 & 53.50 & 218.31 \\
\hline & 1.25 & 15.75 & 11.55 & 19.41 & 17.42 & 15.56 & 22.96 & 9.05 & 16.92 \\
\hline & 1.5 & 5.77 & 4.06 & 6.14 & 4.71 & 4.15 & 4.95 & 3.18 & 3.64 \\
\hline & 2 & 2.41 & 1.57 & 2.44 & 1.60 & 1.70 & 1.38 & 1.56 & 1.08 \\
\hline 0.2 & 0.25 & 3.28 & 0.46 & 3.02 & 0.99 & 1.01 & 0.20 & 1.01 & 0.11 \\
\hline & 0.5 & 6.25 & 1.54 & 5.49 & 1.55 & 4.91 & 6.96 & 3.23 & 4.52 \\
\hline & 0.75 & 17.78 & 7.29 & 22.72 & 17.57 & 92.69 & 127.58 & 54.86 & 224.65 \\
\hline & 1.25 & 15.90 & 11.70 & 19.87 & 17.93 & 16.04 & 23.51 & 9.27 & 17.53 \\
\hline & 1.5 & 5.88 & 4.13 & 6.31 & 4.86 & 4.29 & 5.20 & 3.27 & 3.86 \\
\hline & 2 & 2.49 & 1.62 & 2.52 & 1.68 & 1.75 & 1.47 & 1.59 & 1.14 \\
\hline
\end{tabular}


Table 6. ARL $(\tau)$ and $\operatorname{SDRL}(\tau)$ values of the SSMGR CV-ME chart in the presence of measurement errors for various values of $\tau, \gamma_{0}$

and $\eta$ when $n=5, m=1, B=1$ and $\theta=0$

\begin{tabular}{|c|c|c|c|c|c|c|c|c|c|c|c|c|c|c|c|c|c|c|c|c|c|c|c|}
\hline \multirow[b]{2}{*}{$\gamma_{0}$} & \multirow[b]{2}{*}{$\tau$} & \multicolumn{2}{|c|}{$\eta=0$} & \multicolumn{5}{|c|}{$\eta=0.1$} & \multicolumn{5}{|c|}{$\eta=0.3$} & \multicolumn{5}{|c|}{$\eta=0.5$} & \multicolumn{5}{|c|}{$\eta=1$} \\
\hline & & $\operatorname{ARL}(\tau)$ & $\operatorname{SDRL}(\tau)$ & $k^{*}$ & $C_{1}^{*}$ & $C_{2}^{*}$ & $\operatorname{ARL}(\tau)$ & $\operatorname{SDRL}(\tau)$ & $k^{*}$ & $C_{1}^{*}$ & $C_{2}^{*}$ & $\operatorname{ARL}(\tau)$ & $\operatorname{SDRL}(\tau)$ & $k^{*}$ & $C_{1}^{*}$ & $C_{2}^{*}$ & $\operatorname{ARL}(\tau)$ & $\operatorname{SDRL}(\tau)$ & $k^{*}$ & $C_{1}^{*}$ & $C_{2}^{*}$ & $\operatorname{ARL}(\tau)$ & $\operatorname{SDRL}(\tau)$ \\
\hline \multirow[t]{6}{*}{0.05} & 0.25 & 1.01 & 0.10 & 0.1359 & 1 & 2 & 1.01 & 0.10 & 0.1359 & 1 & 2 & 1.01 & 0.10 & 0.1359 & 1 & 2 & 1.01 & 0.10 & 0.1359 & 1 & 2 & 1.01 & 0.10 \\
\hline & 0.5 & 3.12 & 4.25 & 0.0843 & 1 & 7 & 3.12 & 4.25 & 0.0843 & 1 & 7 & 3.12 & 4.25 & 0.0843 & 1 & 7 & 3.12 & 4.26 & 0.0843 & 1 & 7 & 3.13 & 4.27 \\
\hline & 0.75 & 51.99 & 210.20 & 0.0254 & 1 & 92 & 51.99 & 210.21 & 0.0254 & 1 & 92 & 52.00 & 210.29 & 0.0254 & 1 & 92 & 52.03 & 210.43 & 0.0254 & 1 & 92 & 52.18 & 211.10 \\
\hline & 1.25 & 8.79 & 16.22 & 0.0430 & 1 & 33 & 8.80 & 16.22 & 0.0430 & 1 & 33 & 8.80 & 16.23 & 0.0430 & 1 & 33 & 8.80 & 16.25 & 0.0430 & 1 & 33 & 8.83 & 16.35 \\
\hline & 1.5 & 3.09 & 3.42 & 0.0701 & 1 & 11 & 3.09 & 3.42 & 0.0701 & 1 & 11 & 3.09 & 3.42 & 0.0701 & 1 & 11 & 3.09 & 3.42 & 0.0701 & 1 & 11 & 3.10 & 3.44 \\
\hline & 2 & 1.52 & 1.00 & 0.0962 & 1 & 5 & 1.52 & 1.00 & 0.0962 & 1 & 5 & 1.52 & 1.00 & 0.0962 & 1 & 5 & 1.52 & 1.01 & 0.0962 & 1 & 5 & 1.52 & 1.01 \\
\hline \multirow[t]{6}{*}{0.1} & 0.25 & 1.01 & 0.10 & 0.1359 & 1 & 2 & 1.01 & 0.10 & 0.1359 & 1 & 2 & 1.01 & 0.10 & 0.1359 & 1 & 2 & 1.01 & 0.10 & 0.1359 & 1 & 2 & 1.01 & 0.10 \\
\hline & 0.5 & 3.14 & 4.30 & 0.0843 & 1 & 7 & 3.14 & 4.31 & 0.0843 & 1 & 7 & 3.14 & 4.31 & 0.0843 & 1 & 7 & 3.15 & 4.32 & 0.0843 & 1 & 7 & 3.17 & 4.38 \\
\hline & 0.75 & 52.57 & 212.90 & 0.0254 & 1 & 92 & 52.57 & 212.93 & 0.0254 & 1 & 92 & 52.64 & 213.22 & 0.0254 & 1 & 92 & 52.76 & 213.80 & 0.0251 & 1 & 94 & 53.31 & 217.40 \\
\hline & 1.25 & 8.89 & 16.60 & 0.0430 & 1 & 33 & 8.89 & 16.61 & 0.0430 & 1 & 33 & 8.90 & 16.65 & 0.0424 & 1 & 34 & 8.92 & 16.70 & 0.0424 & 1 & 34 & 9.01 & 16.79 \\
\hline & 1.5 & 3.13 & 3.50 & 0.0701 & 1 & 11 & 3.13 & 3.50 & 0.0701 & 1 & 11 & 3.13 & 3.51 & 0.0701 & 1 & 11 & 3.14 & 3.53 & 0.0701 & 1 & 11 & 3.17 & 3.61 \\
\hline & 2 & 1.53 & 1.03 & 0.0962 & 1 & 5 & 1.53 & 1.03 & 0.0962 & 1 & 5 & 1.54 & 1.03 & 0.0962 & 1 & 5 & 1.54 & 1.04 & 0.0962 & 1 & 5 & 1.55 & 1.07 \\
\hline \multirow[t]{6}{*}{0.15} & 0.25 & 1.01 & 0.10 & 0.1359 & 1 & 2 & 1.01 & 0.10 & 0.1359 & 1 & 2 & 1.01 & 0.10 & 0.1359 & 1 & 2 & 1.01 & 0.11 & 0.1359 & 1 & 2 & 1.01 & 0.11 \\
\hline & 0.5 & 3.18 & 4.39 & 0.0843 & 1 & 7 & 3.18 & 4.40 & 0.0843 & 1 & 7 & 3.18 & 4.41 & 0.0843 & 1 & 7 & 3.19 & 4.44 & 0.0843 & 1 & 7 & 3.24 & 4.56 \\
\hline & 0.75 & 53.50 & 218.31 & 0.0251 & 1 & 94 & 53.52 & 218.39 & 0.0251 & 1 & 94 & 53.66 & 219.04 & 0.0251 & 1 & 94 & 53.94 & 220.35 & 0.0251 & 1 & 94 & 55.25 & 226.46 \\
\hline & 1.25 & 9.05 & 16.92 & 0.0424 & 1 & 34 & 9.05 & 16.93 & 0.0424 & 1 & 34 & 9.07 & 17.03 & 0.0424 & 1 & 34 & 9.12 & 17.22 & 0.0418 & 1 & 35 & 9.34 & 17.81 \\
\hline & 1.5 & 3.18 & 3.64 & 0.0701 & 1 & 11 & 3.18 & 3.65 & 0.0701 & 1 & 11 & 3.19 & 3.67 & 0.0701 & 1 & 11 & 3.21 & 3.71 & 0.0676 & 1 & 12 & 3.29 & 3.71 \\
\hline & 2 & 1.56 & 1.08 & 0.0962 & 1 & 5 & 1.56 & 1.08 & 0.0962 & 1 & 5 & 1.56 & 1.08 & 0.0962 & 1 & 5 & 1.57 & 1.10 & 0.0962 & 1 & 5 & 1.61 & 1.16 \\
\hline \multirow[t]{6}{*}{0.2} & 0.25 & 1.01 & 0.11 & 0.1359 & 1 & 2 & 1.01 & 0.11 & 0.1359 & 1 & 2 & 1.01 & 0.11 & 0.1359 & 1 & 2 & 1.01 & 0.11 & 0.1359 & 1 & 2 & 1.01 & 0.12 \\
\hline & 0.5 & 3.23 & 4.52 & 0.0843 & 1 & 7 & 3.23 & 4.53 & 0.0843 & 1 & 7 & 3.24 & 4.55 & 0.0843 & 1 & 7 & 3.26 & 4.60 & 0.0843 & 1 & 7 & 3.35 & 4.82 \\
\hline & 0.75 & 54.86 & 224.65 & 0.0251 & 1 & 94 & 54.89 & 224.79 & 0.0251 & 1 & 94 & 55.14 & 225.95 & 0.0251 & 1 & 94 & 55.64 & 228.27 & 0.0251 & 1 & 94 & 58.01 & 239.14 \\
\hline & 1.25 & 9.27 & 17.53 & 0.0418 & 1 & 35 & 9.28 & 17.55 & 0.0418 & 1 & 35 & 9.32 & 17.73 & 0.0418 & 1 & 35 & 9.41 & 18.10 & 0.0407 & 1 & 37 & 9.83 & 19.19 \\
\hline & 1.5 & 3.27 & 3.86 & 0.0701 & 1 & 11 & 3.27 & 3.86 & 0.0676 & 1 & 12 & 3.29 & 3.70 & 0.0676 & 1 & 12 & 3.32 & 3.77 & 0.0676 & 1 & 12 & 3.47 & 4.15 \\
\hline & 2 & 1.59 & 1.14 & 0.0962 & 1 & 5 & 1.60 & 1.14 & 0.0962 & 1 & 5 & 1.60 & 1.16 & 0.0962 & 1 & 5 & 1.62 & 1.18 & 0.0962 & 1 & 5 & 1.68 & 1.31 \\
\hline
\end{tabular}


Table 7. ARL $(\tau)$ and $\operatorname{SDRL}(\tau)$ values of the SSMGR CV-ME chart in the presence of measurement errors for various values of $\tau, \gamma_{0}$

and $B$ when $n=5, \eta=0.28, m=1$ and $\theta=0.01$

\begin{tabular}{|c|c|c|c|c|c|c|c|c|c|c|c|c|c|c|c|c|c|c|c|c|c|}
\hline \multirow[b]{2}{*}{$\gamma_{0}$} & \multirow[b]{2}{*}{$\tau$} & \multicolumn{5}{|c|}{$B=1$} & \multicolumn{5}{|c|}{$B=2$} & \multicolumn{5}{|c|}{$B=3$} & \multicolumn{5}{|c|}{$B=4$} \\
\hline & & $k^{*}$ & $C_{1}^{*}$ & $C_{2}^{*}$ & $\operatorname{ARL}(\tau)$ & $\operatorname{SDRL}(\tau)$ & $k^{*}$ & $C_{1}^{*}$ & $C_{2}^{*}$ & $\operatorname{ARL}(\tau)$ & $\operatorname{SDRL}(\tau)$ & $k^{*}$ & $C_{1}^{*}$ & $C_{2}^{*}$ & $\operatorname{ARL}(\tau)$ & $\operatorname{SDRL}(\tau)$ & $k^{*}$ & $C_{1}^{*}$ & $C_{2}^{*}$ & $\operatorname{ARL}(\tau)$ & $\operatorname{SDRL}(\tau)$ \\
\hline \multirow[t]{6}{*}{0.05} & 0.25 & 0.1359 & 1 & 2 & 1.01 & 0.11 & 0.1359 & 1 & 2 & 1.01 & 0.10 & 0.1359 & 1 & 2 & 1.01 & 0.10 & 0.1359 & 1 & 2 & 1.01 & 0.10 \\
\hline & 0.5 & 0.0843 & 1 & 7 & 3.18 & 4.41 & 0.0843 & 1 & 7 & 3.15 & 4.33 & 0.0843 & 1 & 7 & 3.14 & 4.30 & 0.0843 & 1 & 7 & 3.13 & 4.29 \\
\hline & 0.75 & 0.0251 & 1 & 94 & 53.45 & 218.08 & 0.0254 & 1 & 92 & 52.73 & 213.65 & 0.0254 & 1 & 92 & 52.48 & 212.50 & 0.0254 & 1 & 92 & 52.36 & 211.92 \\
\hline & 1.25 & 0.0424 & 1 & 34 & 8.98 & 16.64 & 0.0430 & 1 & 33 & 8.89 & 16.60 & 0.0430 & 1 & 33 & 8.86 & 16.47 & 0.0430 & 1 & 33 & 8.84 & 16.41 \\
\hline & 1.5 & 0.0701 & 1 & 11 & 3.15 & 3.57 & 0.0701 & 1 & 11 & 3.12 & 3.49 & 0.0701 & 1 & 11 & 3.11 & 3.47 & 0.0701 & 1 & 11 & 3.11 & 3.45 \\
\hline & 2 & 0.0962 & 1 & 5 & 1.54 & 1.04 & 0.0962 & 1 & 5 & 1.53 & 1.02 & 0.0962 & 1 & 5 & 1.53 & 1.02 & 0.0962 & 1 & 5 & 1.52 & 1.01 \\
\hline \multirow[t]{6}{*}{0.1} & 0.25 & 0.1359 & 1 & 2 & 1.01 & 0.11 & 0.1359 & 1 & 2 & 1.01 & 0.10 & 0.1359 & 1 & 2 & 1.01 & 0.10 & 0.1359 & 1 & 2 & 1.01 & 0.10 \\
\hline & 0.5 & 0.0843 & 1 & 7 & 3.21 & 4.47 & 0.0843 & 1 & 7 & 3.17 & 4.39 & 0.0843 & 1 & 7 & 3.16 & 4.36 & 0.0843 & 1 & 7 & 3.16 & 4.35 \\
\hline & 0.75 & 0.0251 & 1 & 94 & 54.08 & 221.01 & 0.0251 & 1 & 94 & 53.29 & 217.31 & 0.0254 & 1 & 92 & 53.07 & 215.22 & 0.0254 & 1 & 92 & 52.94 & 214.63 \\
\hline & 1.25 & 0.0424 & 1 & 34 & 9.08 & 17.06 & 0.0424 & 1 & 34 & 8.98 & 16.65 & 0.0424 & 1 & 34 & 8.95 & 16.52 & 0.0424 & 1 & 34 & 8.93 & 16.46 \\
\hline & 1.5 & 0.0701 & 1 & 11 & 3.19 & 3.66 & 0.0701 & 1 & 11 & 3.16 & 3.58 & 0.0701 & 1 & 11 & 3.15 & 3.55 & 0.0701 & 1 & 11 & 3.14 & 3.54 \\
\hline & 2 & 0.0962 & 1 & 5 & 1.56 & 1.07 & 0.0962 & 1 & 5 & 1.55 & 1.05 & 0.0962 & 1 & 5 & 1.54 & 1.04 & 0.0962 & 1 & 5 & 1.54 & 1.04 \\
\hline \multirow[t]{6}{*}{0.15} & 0.25 & 0.1359 & 1 & 2 & 1.01 & 0.11 & 0.1359 & 1 & 2 & 1.01 & 0.11 & 0.1359 & 1 & 2 & 1.01 & 0.11 & 0.1359 & 1 & 2 & 1.01 & 0.11 \\
\hline & 0.5 & 0.0843 & 1 & 7 & 3.25 & 4.57 & 0.0843 & 1 & 7 & 3.21 & 4.48 & 0.0843 & 1 & 7 & 3.20 & 4.45 & 0.0843 & 1 & 7 & 3.19 & 4.44 \\
\hline & 0.75 & 0.0251 & 1 & 94 & 55.14 & 225.91 & 0.0251 & 1 & 94 & 54.28 & 221.94 & 0.0251 & 1 & 94 & 54.01 & 220.69 & 0.0251 & 1 & 94 & 53.88 & 220.08 \\
\hline & 1.25 & 0.0418 & 1 & 35 & 9.25 & 17.43 & 0.0418 & 1 & 35 & 9.14 & 16.98 & 0.0424 & 1 & 34 & 9.11 & 17.18 & 0.0424 & 1 & 34 & 9.09 & 17.11 \\
\hline & 1.5 & 0.0701 & 1 & 11 & 3.25 & 3.82 & 0.0701 & 1 & 11 & 3.22 & 3.73 & 0.0701 & 1 & 11 & 3.20 & 3.70 & 0.0701 & 1 & 11 & 3.20 & 3.68 \\
\hline & 2 & 0.0962 & 1 & 5 & 1.58 & 1.12 & 0.0962 & 1 & 5 & 1.57 & 1.10 & 0.0962 & 1 & 5 & 1.57 & 1.09 & 0.0962 & 1 & 5 & 1.56 & 1.09 \\
\hline \multirow[t]{6}{*}{0.2} & 0.25 & 0.1359 & 1 & 2 & 1.01 & 0.11 & 0.1359 & 1 & 2 & 1.01 & 0.11 & 0.1359 & 1 & 2 & 1.01 & 0.11 & 0.1359 & 1 & 2 & 1.01 & 0.11 \\
\hline & 0.5 & 0.0843 & 1 & 7 & 3.30 & 4.71 & 0.0843 & 1 & 7 & 3.26 & 4.61 & 0.0843 & 1 & 7 & 3.25 & 4.58 & 0.0843 & 1 & 7 & 3.25 & 4.57 \\
\hline & 0.75 & 0.0251 & 1 & 94 & 56.62 & 232.77 & 0.0251 & 1 & 94 & 55.68 & 228.42 & 0.0251 & 1 & 94 & 55.39 & 227.10 & 0.0251 & 1 & 94 & 55.25 & 226.46 \\
\hline & 1.25 & 0.0412 & 1 & 36 & 9.50 & 18.13 & 0.0418 & 1 & 35 & 9.37 & 17.95 & 0.0418 & 1 & 35 & 9.34 & 17.80 & 0.0418 & 1 & 35 & 9.32 & 17.73 \\
\hline & 1.5 & 0.0676 & 1 & 12 & 3.34 & 3.84 & 0.0676 & 1 & 12 & 3.30 & 3.74 & 0.0676 & 1 & 12 & 3.29 & 3.71 & 0.0676 & 1 & 12 & 3.28 & 3.69 \\
\hline & 2 & 0.0962 & 1 & 5 & 1.62 & 1.20 & 0.0962 & 1 & 5 & 1.61 & 1.17 & 0.0962 & 1 & 5 & 1.60 & 1.16 & 0.0962 & 1 & 5 & 1.60 & 1.15 \\
\hline
\end{tabular}


Table 8. ARL $(\tau)$ and $\operatorname{SDRL}(\tau)$ values of the SSMGR CV-ME chart in the presence of measurement errors for various values of $\tau, \gamma_{0}$ and $m$ when $n=5, \eta=0.28, B=1$ and $\theta=0.01$

\begin{tabular}{|c|c|c|c|c|c|c|c|c|c|c|c|c|c|c|c|c|c|c|c|c|c|}
\hline \multirow[b]{2}{*}{$\gamma_{0}$} & \multirow[b]{2}{*}{$\tau$} & \multicolumn{5}{|c|}{$m=1$} & \multicolumn{5}{|c|}{$m=3$} & \multicolumn{5}{|c|}{$m=5$} & \multicolumn{5}{|c|}{$m=7$} \\
\hline & & $k^{*}$ & $C_{1}^{*}$ & $C_{2}^{*}$ & $\operatorname{ARL}(\tau)$ & $\operatorname{SDRL}(\tau)$ & $k^{*}$ & $C_{1}^{*}$ & $C_{2}^{*}$ & $\operatorname{ARL}(\tau)$ & $\operatorname{SDRL}(\tau)$ & $k^{*}$ & $C_{1}^{*}$ & $C_{2}^{*}$ & $\operatorname{ARL}(\tau)$ & $\operatorname{SDRL}(\tau)$ & $k^{*}$ & $C_{1}^{*}$ & $C_{2}^{*}$ & $\operatorname{ARL}(\tau)$ & $\operatorname{SDRL}(\tau)$ \\
\hline \multirow[t]{5}{*}{0.05} & 0.25 & 0.1359 & 1 & 2 & 1.01 & 0.11 & 0.1359 & 1 & 2 & 1.01 & 0.11 & 0.1359 & 1 & 2 & 1.01 & 0.11 & 0.1359 & 1 & 2 & 1.01 & 0.11 \\
\hline & 0.5 & 0.0843 & 1 & 7 & 3.18 & 4.41 & 0.0843 & 1 & 7 & 3.18 & 4.41 & 0.0843 & 1 & 7 & 3.18 & 4.41 & 0.0843 & 1 & 7 & 3.18 & 4.41 \\
\hline & 0.75 & 0.0251 & 1 & 94 & 53.45 & 218.08 & 0.0251 & 1 & 94 & 53.44 & 218.03 & 0.0251 & 1 & 94 & 53.44 & 218.02 & 0.0251 & 1 & 94 & 53.44 & 218.01 \\
\hline & 1.25 & 0.0424 & 1 & 34 & 8.98 & 16.64 & 0.0424 & 1 & 34 & 8.98 & 16.64 & 0.0424 & 1 & 34 & 8.98 & 16.64 & 0.0424 & 1 & 34 & 8.98 & 16.64 \\
\hline & 1.5 & 0.0701 & 1 & 11 & 3.15 & 3.57 & 0.0701 & 1 & 11 & 3.15 & 3.57 & 0.0701 & 1 & 11 & 3.15 & 3.57 & 0.0701 & 1 & 11 & 3.15 & 3.57 \\
\hline \multirow[t]{6}{*}{0.1} & 0.25 & 0.1359 & 1 & 2 & 1.01 & 0.11 & 0.1359 & 1 & 2 & 1.01 & 0.11 & 0.1359 & 1 & 2 & 1.01 & 0.11 & 0.1359 & 1 & 2 & 1.01 & 0.11 \\
\hline & 0.5 & 0.0843 & 1 & 7 & 3.21 & 4.47 & 0.0843 & 1 & 7 & 3.21 & 4.47 & 0.0843 & 1 & 7 & 3.21 & 4.47 & 0.0843 & 1 & 7 & 3.21 & 4.47 \\
\hline & 0.75 & 0.0251 & 1 & 94 & 54.08 & 221.01 & 0.0251 & 1 & 94 & 54.04 & 220.82 & 0.0251 & 1 & 94 & 54.03 & 220.79 & 0.0251 & 1 & 94 & 54.03 & 220.77 \\
\hline & 1.25 & 0.0424 & 1 & 34 & 9.08 & 17.06 & 0.0424 & 1 & 34 & 9.07 & 17.03 & 0.0424 & 1 & 34 & 9.07 & 17.03 & 0.0424 & 1 & 34 & 9.07 & 17.02 \\
\hline & 1.5 & 0.0701 & 1 & 11 & 3.19 & 3.66 & 0.0701 & 1 & 11 & 3.19 & 3.65 & 0.0701 & 1 & 11 & 3.19 & 3.65 & 0.0701 & 1 & 11 & 3.19 & 3.65 \\
\hline & 2 & 0.0962 & 1 & 5 & 1.56 & 1.07 & 0.0962 & 1 & 5 & 1.56 & 1.07 & 0.0962 & 1 & 5 & 1.56 & 1.07 & 0.0962 & 1 & 5 & 1.56 & 1.07 \\
\hline \multirow[t]{6}{*}{0.15} & 0.25 & 0.1359 & 1 & 2 & 1.01 & 0.11 & 0.1359 & 1 & 2 & 1.01 & 0.11 & 0.1359 & 1 & 2 & 1.01 & 0.11 & 0.1359 & 1 & 2 & 1.01 & 0.11 \\
\hline & 0.5 & 0.0843 & 1 & 7 & 3.25 & 4.57 & 0.0843 & 1 & 7 & 3.24 & 4.56 & 0.0843 & 1 & 7 & 3.24 & 4.56 & 0.0843 & 1 & 7 & 3.24 & 4.56 \\
\hline & 0.75 & 0.0251 & 1 & 94 & 55.14 & 225.91 & 0.0251 & 1 & 94 & 55.04 & 225.49 & 0.0251 & 1 & 94 & 55.03 & 225.40 & 0.0251 & 1 & 94 & 55.02 & 225.37 \\
\hline & 1.25 & 0.0418 & 1 & 35 & 9.25 & 17.43 & 0.0418 & 1 & 35 & 9.23 & 17.37 & 0.0418 & 1 & 35 & 9.23 & 17.35 & 0.0418 & 1 & 35 & 9.23 & 17.35 \\
\hline & 1.5 & 0.0701 & 1 & 11 & 3.25 & 3.82 & 0.0701 & 1 & 11 & 3.25 & 3.80 & 0.0701 & 1 & 11 & 3.25 & 3.80 & 0.0701 & 1 & 11 & 3.25 & 3.80 \\
\hline & 2 & 0.0962 & 1 & 5 & 1.58 & 1.12 & 0.0962 & 1 & 5 & 1.58 & 1.12 & 0.0962 & 1 & 5 & 1.58 & 1.12 & 0.0962 & 1 & 5 & 1.58 & 1.12 \\
\hline \multirow[t]{6}{*}{0.2} & 0.25 & 0.1359 & 1 & 2 & 1.01 & 0.11 & 0.1359 & 1 & 2 & 1.01 & 0.11 & 0.1359 & 1 & 2 & 1.01 & 0.11 & 0.1359 & 1 & 2 & 1.01 & 0.11 \\
\hline & 0.5 & 0.0843 & 1 & 7 & 3.30 & 4.71 & 0.0843 & 1 & 7 & 3.30 & 4.70 & 0.0843 & 1 & 7 & 3.30 & 4.69 & 0.0843 & 1 & 7 & 3.30 & 4.69 \\
\hline & 0.75 & 0.0251 & 1 & 94 & 56.62 & 232.77 & 0.0251 & 1 & 94 & 56.46 & 232.01 & 0.0251 & 1 & 94 & 56.42 & 231.86 & 0.0251 & 1 & 94 & 56.41 & 231.80 \\
\hline & 1.25 & 0.0412 & 1 & 36 & 9.50 & 18.13 & 0.0412 & 1 & 36 & 9.47 & 18.01 & 0.0412 & 1 & 36 & 9.46 & 17.99 & 0.0412 & 1 & 36 & 9.46 & 17.98 \\
\hline & 1.5 & 0.0676 & 1 & 12 & 3.34 & 3.84 & 0.0676 & 1 & 12 & 3.33 & 3.81 & 0.0676 & 1 & 12 & 3.33 & 3.81 & 0.0676 & 1 & 12 & 3.33 & 3.81 \\
\hline & 2 & 0.0962 & 1 & 5 & 1.62 & 1.20 & 0.0962 & 1 & 5 & 1.62 & 1.19 & 0.0962 & 1 & 5 & 1.62 & 1.19 & 0.0962 & 1 & 5 & 1.62 & 1.19 \\
\hline
\end{tabular}


Table 9. ARL $(\tau)$ and $\operatorname{SDRL}(\tau)$ values of the SSMGR CV-ME chart in the presence of measurement errors for various values of $\tau, \gamma_{0}$ and $\theta$ when $n=5, \eta=0.28, B=1$ and $m=1$

\begin{tabular}{|c|c|c|c|c|c|c|c|c|c|c|c|c|c|c|c|c|c|c|c|c|c|}
\hline \multirow[b]{2}{*}{$\gamma_{0}$} & \multirow[b]{2}{*}{$\tau$} & \multicolumn{5}{|c|}{$\theta=0$} & \multicolumn{5}{|c|}{$\theta=0.01$} & \multicolumn{5}{|c|}{$\theta=0.03$} & \multicolumn{5}{|c|}{$\theta=0.05$} \\
\hline & & $k^{*}$ & $C_{1}^{*}$ & $C_{2}^{*}$ & $\operatorname{ARL}(\tau)$ & $\operatorname{SDRL}(\tau)$ & $k^{*}$ & $C_{1}^{*}$ & $C_{2}^{*}$ & $\operatorname{ARL}(\tau)$ & $\operatorname{SDRL}(\tau)$ & $k^{*}$ & $C_{1}^{*}$ & $C_{2}^{*}$ & $\operatorname{ARL}(\tau)$ & $\operatorname{SDRL}(\tau)$ & $k^{*}$ & $C_{1}^{*}$ & $C_{2}^{*}$ & $\operatorname{ARL}(\tau)$ & $\operatorname{SDRL}(\tau)$ \\
\hline \multirow[t]{6}{*}{0.05} & 0.25 & 0.1359 & 1 & 2 & 1.01 & 0.10 & 0.1359 & 1 & 2 & 1.01 & 0.11 & 0.1359 & 1 & 2 & 1.01 & 0.12 & 0.1359 & 1 & 2 & 1.01 & 0.13 \\
\hline & 0.5 & 0.0843 & 1 & 7 & 3.12 & 4.25 & 0.0843 & 1 & 7 & 3.18 & 4.41 & 0.0843 & 1 & 7 & 3.32 & 4.75 & 0.0799 & 1 & 8 & 3.46 & 4.91 \\
\hline & 0.75 & 0.0254 & 1 & 92 & 52.00 & 210.27 & 0.0251 & 1 & 94 & 53.45 & 218.08 & 0.0251 & 1 & 94 & 56.50 & 232.21 & 0.0248 & 1 & 96 & 59.63 & 247.86 \\
\hline & 1.25 & 0.0430 & 1 & 33 & 8.80 & 16.23 & 0.0424 & 1 & 34 & 8.98 & 16.64 & 0.0418 & 1 & 35 & 9.35 & 17.85 & 0.0407 & 1 & 37 & 9.73 & 18.77 \\
\hline & 1.5 & 0.0701 & 1 & 11 & 3.09 & 3.42 & 0.0701 & 1 & 11 & 3.15 & 3.57 & 0.0676 & 1 & 12 & 3.28 & 3.68 & 0.0676 & 1 & 12 & 3.40 & 3.99 \\
\hline & 2 & 0.0962 & 1 & 5 & 1.52 & 1.00 & 0.0962 & 1 & 5 & 1.54 & 1.04 & 0.0962 & 1 & 5 & 1.59 & 1.13 & 0.0962 & 1 & 5 & 1.63 & 1.22 \\
\hline \multirow[t]{6}{*}{0.1} & 0.25 & 0.1359 & 1 & 2 & 1.01 & 0.10 & 0.1359 & 1 & 2 & 1.01 & 0.11 & 0.1359 & 1 & 2 & 1.01 & 0.12 & 0.1359 & 1 & 2 & 1.01 & 0.13 \\
\hline & 0.5 & 0.0843 & 1 & 7 & 3.14 & 4.31 & 0.0843 & 1 & 7 & 3.21 & 4.47 & 0.0843 & 1 & 7 & 3.34 & 4.81 & 0.0799 & 1 & 8 & 3.48 & 4.98 \\
\hline & 0.75 & 0.0254 & 1 & 92 & 52.63 & 213.18 & 0.0251 & 1 & 94 & 54.08 & 221.01 & 0.0251 & 1 & 94 & 57.14 & 235.17 & 0.0248 & 1 & 96 & 60.28 & 250.85 \\
\hline & 1.25 & 0.0430 & 1 & 33 & 8.90 & 16.65 & 0.0424 & 1 & 34 & 9.08 & 17.06 & 0.0412 & 1 & 36 & 9.45 & 17.93 & 0.0407 & 1 & 37 & 9.82 & 19.20 \\
\hline & 1.5 & 0.0701 & 1 & 11 & 3.13 & 3.51 & 0.0701 & 1 & 11 & 3.19 & 3.66 & 0.0676 & 1 & 12 & 3.31 & 3.77 & 0.0676 & 1 & 12 & 3.44 & 4.09 \\
\hline & 2 & 0.0962 & 1 & 5 & 1.54 & 1.03 & 0.0962 & 1 & 5 & 1.56 & 1.07 & 0.0962 & 1 & 5 & 1.60 & 1.16 & 0.0962 & 1 & 5 & 1.65 & 1.25 \\
\hline \multirow[t]{6}{*}{0.15} & 0.25 & 0.1359 & 1 & 2 & 1.01 & 0.10 & 0.1359 & 1 & 2 & 1.01 & 0.11 & 0.1359 & 1 & 2 & 1.01 & 0.12 & 0.1359 & 1 & 2 & 1.01 & 0.13 \\
\hline & 0.5 & 0.0843 & 1 & 7 & 3.18 & 4.41 & 0.0843 & 1 & 7 & 3.25 & 4.57 & 0.0843 & 1 & 7 & 3.39 & 4.91 & 0.0799 & 1 & 8 & 3.53 & 5.08 \\
\hline & 0.75 & 0.0251 & 1 & 94 & 53.64 & 218.95 & 0.0251 & 1 & 94 & 55.14 & 225.91 & 0.0251 & 1 & 94 & 58.22 & 240.09 & 0.0248 & 1 & 96 & 61.38 & 255.84 \\
\hline & 1.25 & 0.0424 & 1 & 34 & 9.07 & 17.01 & 0.0418 & 1 & 35 & 9.25 & 17.43 & 0.0412 & 1 & 36 & 9.62 & 18.65 & 0.0401 & 1 & 38 & 9.99 & 19.58 \\
\hline & 1.5 & 0.0701 & 1 & 11 & 3.19 & 3.66 & 0.0701 & 1 & 11 & 3.25 & 3.82 & 0.0676 & 1 & 12 & 3.38 & 3.92 & 0.0676 & 1 & 12 & 3.50 & 4.25 \\
\hline & 2 & 0.0962 & 1 & 5 & 1.56 & 1.08 & 0.0962 & 1 & 5 & 1.58 & 1.12 & 0.0962 & 1 & 5 & 1.63 & 1.21 & 0.0962 & 1 & 5 & 1.68 & 1.31 \\
\hline \multirow[t]{6}{*}{0.2} & 0.25 & 0.1359 & 1 & 2 & 1.01 & 0.11 & 0.1359 & 1 & 2 & 1.01 & 0.11 & 0.1359 & 1 & 2 & 1.01 & 0.12 & 0.1359 & 1 & 2 & 1.02 & 0.14 \\
\hline & 0.5 & 0.0843 & 1 & 7 & 3.24 & 4.55 & 0.0843 & 1 & 7 & 3.30 & 4.71 & 0.0799 & 1 & 8 & 3.44 & 4.87 & 0.0799 & 1 & 8 & 3.58 & 5.23 \\
\hline & 0.75 & 0.0251 & 1 & 94 & 55.11 & 225.79 & 0.0251 & 1 & 94 & 56.62 & 232.77 & 0.0248 & 1 & 96 & 59.70 & 248.17 & 0.0248 & 1 & 96 & 62.91 & 262.81 \\
\hline & 1.25 & 0.0418 & 1 & 35 & 9.31 & 17.70 & 0.0412 & 1 & 36 & 9.50 & 18.13 & 0.0407 & 1 & 37 & 9.86 & 19.36 & 0.0396 & 1 & 39 & 10.24 & 20.30 \\
\hline & 1.5 & 0.0676 & 1 & 12 & 3.28 & 3.69 & 0.0676 & 1 & 12 & 3.34 & 3.84 & 0.0676 & 1 & 12 & 3.47 & 4.15 & 0.0653 & 1 & 13 & 3.59 & 4.27 \\
\hline & 2 & 0.0962 & 1 & 5 & 1.60 & 1.16 & 0.0962 & 1 & 5 & 1.62 & 1.20 & 0.0962 & 1 & 5 & 1.67 & 1.29 & 0.0962 & 1 & 5 & 1.72 & 1.39 \\
\hline
\end{tabular}


Table 10. Phase-I and Phase-II datasets on the weight of scrap zinc alloy material in a die casting hot chamber process

\begin{tabular}{cccccccc}
\hline & \multicolumn{3}{c}{ Phase-I } & & \multicolumn{3}{c}{ Phase-II } \\
\cline { 2 - 3 } \cline { 6 - 7 }$i$ & $\bar{X}_{i}$ & $S_{i}$ & $\hat{\gamma}_{i}$ & & $\bar{X}_{i}$ & $S_{i}$ & $\hat{\gamma}_{i}$ \\
\hline 1 & 292.6 & 2.701 & 0.0092 & & 396.4 & 4.037 & 0.0102 \\
2 & 289.0 & 0.707 & 0.0024 & & 393.2 & 1.923 & 0.0049 \\
3 & 291.4 & 2.073 & 0.0071 & & 404.6 & 3.049 & 0.0075 \\
4 & 288.0 & 3.937 & 0.0137 & & 396.0 & 2.449 & 0.0062 \\
5 & 290.0 & 0.707 & 0.0024 & & 301.4 & 3.049 & 0.0101 \\
6 & 288.2 & 1.303 & 0.0045 & & 295.4 & 1.816 & 0.0061 \\
7 & 535.4 & 8.264 & 0.0154 & & 293.2 & 1.788 & 0.0061 \\
8 & 518.4 & 7.224 & 0.0139 & & 297.4 & 2.190 & 0.0074 \\
9 & 529.2 & 9.203 & 0.0174 & & 642.8 & 2.280 & $\mathbf{0 . 0 0 3 5}$ \\
10 & 527.0 & 9.591 & 0.0182 & & 640.2 & 1.095 & $\mathbf{0 . 0 0 1 7}$ \\
11 & 533.6 & 4.929 & 0.0092 & & 650.4 & 3.435 & 0.0053 \\
12 & 439.2 & 3.114 & 0.0071 & & 647.8 & 1.643 & $\mathbf{0 . 0 0 2 5}$ \\
13 & 447.2 & 2.774 & 0.0062 & & 646.0 & 2.345 & $\mathbf{0 . 0 0 3 6}$ \\
14 & 443.4 & 8.173 & 0.0184 & & 549.8 & 3.114 & 0.0057 \\
15 & 434.0 & 2.549 & 0.0059 & & 522.6 & 10.310 & $\mathbf{0 . 0 1 9 7}$ \\
16 & 436.0 & 1.224 & 0.0028 & & 519.8 & 7.259 & 0.0140 \\
17 & 437.6 & 2.408 & 0.0055 & & 518.8 & 8.927 & $\mathbf{0 . 0 1 7 2}$ \\
18 & 419.6 & 4.037 & 0.0096 & & 515.4 & 11.760 & $\mathbf{0 . 0 2 2 8}$ \\
19 & 422.4 & 4.159 & 0.0098 & & 550.4 & 15.678 & $\mathbf{0 . 0 2 8 5}$ \\
20 & 416.8 & 3.962 & 0.0095 & & 529.0 & 10.440 & $\mathbf{0 . 0 1 9 7}$ \\
21 & 420.4 & 4.979 & 0.0118 & & 526.8 & 9.602 & $\mathbf{0 . 0 1 8 2}$ \\
22 & 421.6 & 2.302 & 0.0055 & & 529.2 & 7.949 & 0.0150 \\
23 & 418.4 & 4.393 & 0.0105 & & 521.8 & 7.981 & 0.0153 \\
24 & 410.4 & 4.219 & 0.0103 & & 534.0 & 7.681 & 0.0144 \\
25 & 449.0 & 6.204 & 0.0138 & & 525.0 & 5.656 & 0.0108 \\
26 & 441.6 & 3.781 & 0.0086 & & 533.0 & 5.522 & 0.0104 \\
27 & 393.2 & 6.220 & 0.0158 & & 287.8 & 3.114 & 0.0108 \\
28 & 401.8 & 1.483 & 0.0037 & & 287.2 & 3.271 & 0.0114 \\
29 & 412.6 & 3.049 & 0.0074 & & 289.8 & 1.095 & $\mathbf{0 . 0 0 3 8}$ \\
30 & 461.4 & 7.700 & 0.0167 & & 288.4 & 3.049 & 0.0106 \\
\hline & & & & & & &
\end{tabular}




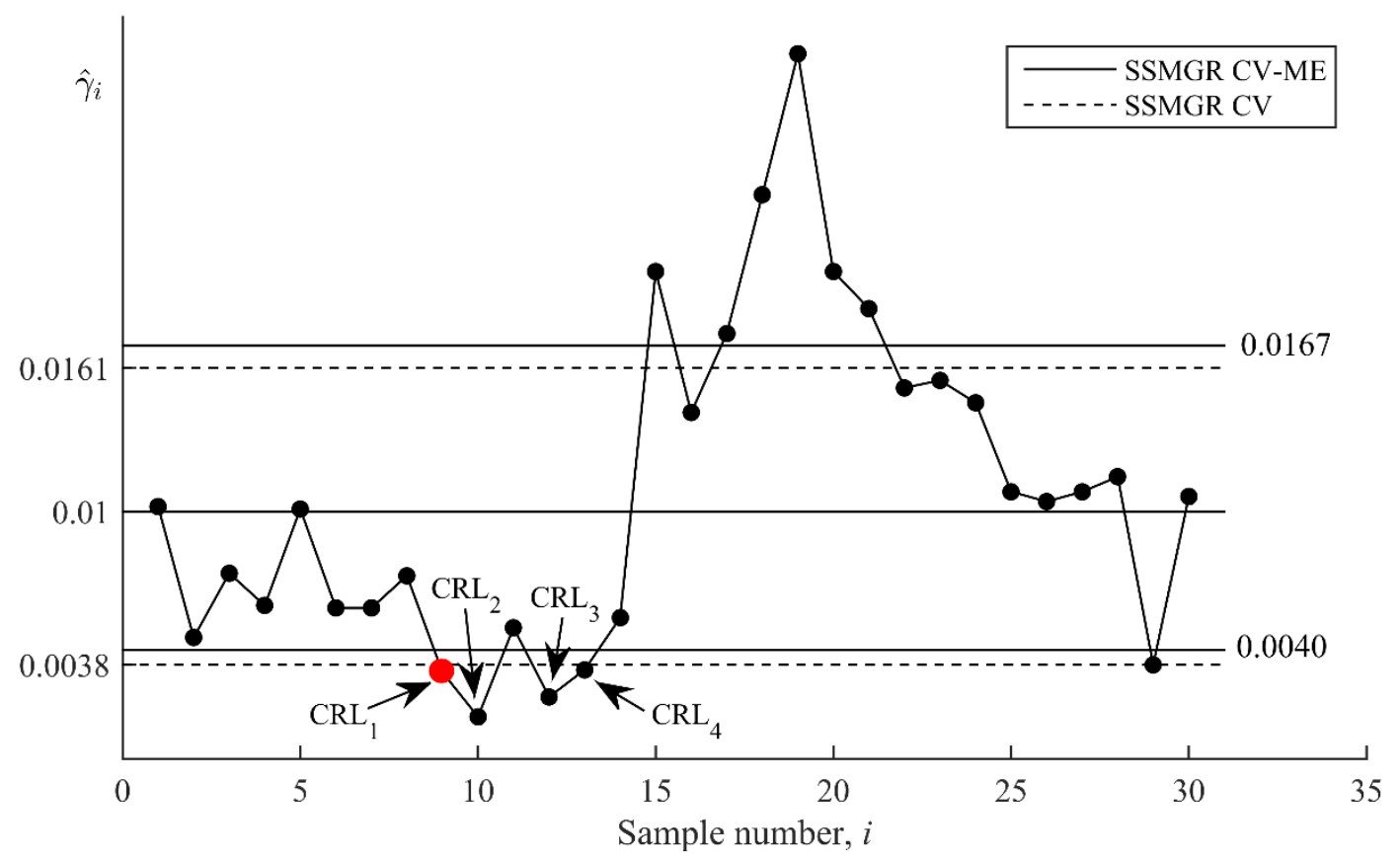

Figure 1. SSMGR CV and SSMGR CV-ME charts for monitoring the Phase-II sample CVs $\left(\hat{\gamma}_{i}\right.$, for $i=1,2, \ldots, 30)$ on the weights of scrap zinc alloy material in a die casting hot chamber process 